\title{
SQUARED HOPF ALGEBRAS AND RECONSTRUCTION THEOREMS
}

\author{
VOLODYMYR V. LYUBASHENKO \\ Mathematical Methods of Systems Analysis \\ Department of Applied Mathematics \\ Kiev Polytechnic Institute \\ 37 prosp. Peremogy, 252056 Kiev, Ukraine
}

\begin{abstract}
Given an abelian $\mathbb{k}$-linear rigid monoidal category $\mathcal{V}$, where $\mathbb{k}$ is a perfect field, we define squared coalgebras as objects of cocompleted $\mathcal{V} \otimes \mathcal{V}$ (Deligne's tensor product of categories) equipped with the appropriate notion of comultiplication. Based on this, (squared) bialgebras and Hopf algebras are defined without use of braiding. If $\mathcal{V}$ is the category of $\mathbb{k}$-vector spaces, squared (co)algebras coincide with conventional ones. If $\mathcal{V}$ is braided, a braided Hopf algebra can be obtained from a squared one.

Reconstruction theorems give equivalence of squared co- (bi-, Hopf) algebras in $\mathcal{V}$ and corresponding fibre functors to $\mathcal{V}$ (which is not the case with the usual definitions). Finally, squared quasitriangular Hopf coalgebra is a solution to the problem of defining quantum groups in braided categories.
\end{abstract}

Introduction. Classical reconstruction theorem (e.g. Saavedra [7, Section 2.3.2.1]) asserts that a $\mathbb{k}$-coalgebra can be reconstructed from the underlying functor from its category of comodules to vector spaces. Saavedra [7, Section 2.6 .3 a)] and later Schauenburg [8] also prove that an essentially small abelian $\mathbb{k}$-linear category equipped with an exact faithful functor $\omega$ to the category of finite dimensional $\mathbb{k}$-vector spaces is equivalent to the category of finite dimensional comodules over some $\mathbb{k}$-coalgebra. A direct attempt to generalise these results replacing the category of vector spaces by an abelian $\mathbb{k}$-linear rigid monoidal category $v$ fails. For instance, the category of comodules over the coalgebra constructed from $\omega$ is bigger than the initial category (precise results are formulated by Pareigis [6]). However, if one modifies the definitions of coalgebras and comodules in a monoidal category, the reconstruction theorem will be recovered. This is the main conclusion of this work.

1991 Mathematics Subject Classification: Primary 16W30, 18D35, Secondary 17B37, 18D10. Research was supported in part by the EPSRC research grant GR/G 42976.

The paper is in final form and no version of it will be published elsewhere. 
The new notion will be called a squared coalgebra. The monoidal version of the reconstruction theorem dictates the definition of a squared bialgebra. Squared Hopf (co)algebras based on $\mathcal{V}$ can be also defined, even if $\mathcal{V}$ is not braided, but satisfies a much weaker condition! If $\mathcal{V}$ is braided, a squared Hopf (co)algebra determines a braided Hopf algebra, but not vice versa. Finally, squared quasitriangular Hopf coalgebra is a solution to the problem of defining quantum groups in braided categories.

All definitions are based on the notion of tensor product of abelian categories given by Deligne [1]. The squared notions (coalgebras, bialgebras, Hopf algebras) are objects of the cocompleted tensor square of the initial category $\mathcal{V}$, whence the terminology. The structure maps - comultiplication, multiplication etc. - are morphisms in tensor powers of $\mathcal{V}$. The associativity and other properties mean equality of two composite morphisms in tensor powers of $\mathcal{V}$.

More precisely, we use the cocompletions of tensor powers of $\mathcal{V}$, where the cocompletion of an abelian $\mathbb{k}$-linear category $\mathcal{A}$ with finite dimensional $\mathbb{k}$-spaces of morphisms always means the category $\hat{\mathcal{A}}=$ ind $-\mathcal{A}$, made of filtered inductive limits of objects of $\mathcal{A}$. If $\mathcal{A}$ is essentially small, $\widehat{\mathcal{A}}$ is equivalent to the category $\underline{\operatorname{Hom}}_{\mathbb{k}, \text { l.e. }}\left(\mathcal{A}^{\text {op }}, \mathbb{k}\right.$-vect) of left exact functors $\mathcal{A}^{\text {op }} \rightarrow \mathbb{k}$-vect. (See Grothendieck and Verdier [3].)

We assume that $\mathbb{k}$ is a perfect field.

Let us recall the reconstruction theorem in details. If $\omega: \mathcal{C} \rightarrow \mathbb{k}$-vect is a faithful exact $\mathbb{k}$-linear functor and $\mathcal{C}$ is essentially small, then there is an equivalence $F$ of $\mathcal{C}$ with the category of $C$-comodules, where $C$ is the $\mathbb{k}$-coalgebra

$$
C=\int^{X \in \mathcal{C}} \omega(X) \otimes_{\mathbb{k}} \omega(X)^{*},
$$

and $\omega$ is isomorphic to the composite of $F$ and the underlying functor $\mathcal{U}: C$-comod $\rightarrow$ $\mathbb{k}$-vect. When $\mathbb{k}$-vect is replaced by an abelian $\mathbb{k}$-linear rigid monoidal category $\mathcal{V}$ with finite dimensional spaces $\operatorname{Hom}_{\mathcal{v}}(-,-)$ such that End $I=\mathbb{k}$ ( $I$ is the unit object) and each object has finite length, a version of reconstruction theorem holds, although $F$ is no longer an equivalence. It turns out that by modifying the definitions of coalgebras and comodules one can make $F$ into an equivalence, thus recovering the original form of the theorem. Namely, instead of the coalgebra in $\widehat{\mathcal{V}}$

$$
\bar{C}=\int^{X \in \mathcal{C}} \omega(X) \otimes \omega(X)^{\vee},
$$

one can use the squared coalgebra

$$
C=\int^{X \in \mathcal{C}} \omega(X) \odot \omega(X)^{\vee} \in \widehat{\mathcal{V} \otimes} \mathcal{V},
$$

where $\odot: \mathcal{V} \times \mathcal{V} \rightarrow \mathcal{V} \otimes \mathcal{V}$ is the canonical functor of exterior tensor product.

To explain the definition of a squared coalgebra, we recall that the tensor product functor $\otimes: \mathcal{V} \times \mathcal{V} \rightarrow \mathcal{V}$ can be decomposed as $\mathcal{V} \times \mathcal{V} \stackrel{\odot}{\rightarrow} \mathcal{V} \otimes \mathcal{V} \stackrel{\circledast}{\longrightarrow} \mathcal{V}$ up to an isomorphism. Using the functor $\odot$ and the diagonal restriction functor $\circledast$ we can construct various objects like $C_{13} \odot I_{2} \in \widehat{\mathcal{Q} \otimes 3}$ ( $I$ is the unit object and the subindices indicate the tensorands to which an object is placed), or like $C_{12^{\prime}} \otimes C_{2^{\prime \prime} 3} \in \widehat{\mathcal{V}^{\otimes 3}}$ (this is the result of applying $\circledast$ 
on the second and third places to $C_{12} \odot C_{34} \in \widehat{\mathcal{V} \otimes 4}$ ), or like $C_{1^{\prime} 1^{\prime \prime}}=\circledast C \in \widehat{\mathcal{V}}$ (the dash and the double dash indicate the order of multiplicands) etc. Notice that $\circledast$ applied to (2) gives (1).

The reader is advised to consider the example $\mathcal{V}=H$-comod (finite dimensional $H$ comodules) throughout this work, where $H$ is a Hopf $\mathbb{k}$-algebra. Then $\widehat{\mathcal{V}}=H$-Comod (all $H$-comodules), $\mathcal{V}^{\otimes n}=H^{\otimes n}$-comod, $\widehat{\mathcal{V} \otimes n}=H^{\otimes n}$-Comod. The functor $\odot: \mathcal{V} \times \mathcal{V} \rightarrow \mathcal{V} \otimes \mathcal{V}$ sends a pair of $H$-comodules $(X, Y)$ to the $H \otimes_{\mathbb{k}} H$-comodule $X \otimes_{\mathbb{k}} Y$ (exterior tensor product of comodules) and the diagonal restriction functor $\circledast: \mathcal{V} \otimes \mathcal{V} \rightarrow \mathcal{V}$ sends an $H \otimes_{\mathbb{k}} H$-comodule to an $H$-comodule via the homomorphism of coalgebras $m: H \otimes_{\mathbb{k}}$ $H \rightarrow H$ - the multiplication. Thus, given an $H \otimes_{\mathbb{k}} H$-comodule $C$ with the coaction $\delta: c \mapsto c_{(1)} \otimes c_{(2)} \otimes c_{(3)} \in H \otimes_{\mathbb{k}} H \otimes_{\mathbb{k}} C$ we get the $H^{\otimes 3}$-comodule $C_{13} \odot I_{2}=(C, \delta: c \mapsto$ $\left.c_{(1)} \otimes 1 \otimes c_{(2)} \otimes c_{(3)} \in H^{\otimes 3} \otimes_{\mathbb{k}} C\right)$, the $H^{\otimes 3}$-comodule $C_{12^{\prime}} \otimes C_{2^{\prime \prime} 3}=\left(C \otimes_{\mathbb{k}} C, c \otimes d \mapsto\right.$ $\left.c_{(1)} \otimes c_{(2)} d_{(1)} \otimes d_{(2)} \otimes c_{(3)} \otimes d_{(3)} \in H^{\otimes 3} \otimes_{\mathbb{k}} C^{\otimes 2}\right)$, the $H$-comodule $\circledast C=(C, \delta: c \mapsto$ $\left.c_{(1)} c_{(2)} \otimes c_{(3)} \in H \otimes_{\mathbb{k}} C\right)$ etc.

Now we may define a squared coalgebra as an object $C \in \widehat{\mathcal{V} \otimes \mathcal{V}}$ equipped with the comultiplication $\Delta_{123}: C_{13} \odot I_{2} \rightarrow C_{12^{\prime}} \otimes C_{2^{\prime \prime} 3}$ and the counit $\varepsilon: C_{1^{\prime} 1^{\prime \prime}} \rightarrow I_{1}$, which satisfy the coassociativity axiom (an equation in $\widehat{\mathcal{V}^{\otimes 4}}$, see (2.1.1)) and two axioms for the counit (equations (2.1.2a)-(2.1.2b) in $\left.\widehat{\mathcal{V}^{2}}\right)$. A $C$-comodule is an object $X \in \mathcal{V}$ equipped with the coaction $\delta: X_{1} \odot I_{2} \rightarrow C_{12^{\prime}} \otimes X_{2^{\prime \prime}} \in \widehat{V^{\otimes 2}}$, which is coassociative (equation (2.2.1) in $\widehat{\nu 3}$ ) and counital (equation $(2.2 .2)$ in $\widehat{\nabla^{2} 2}$ ). It turns out that for any object $Y \in \mathcal{V}$ the object $Y \odot Y^{\vee} \in \mathcal{V} \otimes \mathcal{V}$ has the canonical structure of a squared coalgebra and $Y$ is a comodule over it. We deduce that the coend (2) is a squared coalgebra as well. Moreover, the second part of the reconstruction theorem claims that any squared coalgebra is isomorphic to a coalgebra of the form (2).

Thus, the full form of the reconstruction theorem asserts equivalence of the following two categories: the category of $\mathbb{k}$-linear exact faithful functors from an essentially small category to $\mathcal{V}$ and the category of squared coalgebras in $\mathcal{V}$. Philosophically, categories over the category $\mathcal{V}$ are fully encoded in terms of coalgebras living in $\mathcal{V}$ (in fact, in $\widehat{\mathcal{Q}} \mathcal{V}$ ) and vice versa. Comparing the category of comodules ${ }^{C} \mathcal{V}$ over a squared coalgebra $C$ and the category of comodules ${ }^{\bar{C}} \mathcal{V}$ over the ordinary coalgebra (comonoid) $\bar{C}=\circledast C$ we get $\bar{C} \mathcal{V}={ }^{C} \mathcal{V} \otimes \mathcal{V}$. That is expected from the description of the coend reconstructed from the functor ${ }^{\bar{C}} \mathcal{V} \rightarrow \mathcal{V}$ given by Pareigis [6] in the case $\mathcal{V}=H$-comod for a Hopf algebra $H$.

The monoidal version of the reconstruction theorem also holds. Namely, the category of monoidal $\mathbb{k}$-linear exact faithful functors $\omega: \mathcal{C} \rightarrow \mathcal{V}$ ( $\mathcal{C}$ is essentially small) and the category of squared bicoalgebras in $\mathcal{V}$ are equivalent. A squared bicoalgebra is defined as an object of $\widehat{\mathcal{Q}} \mathcal{V}$ having the structure of a squared coalgebra and of an algebra in the monoidal category $\widehat{\mathcal{\otimes} V}$ with compatibility axioms which require that the multiplication and the unit were homomorphisms of coalgebras. (There are several monoidal structures in $\widehat{\mathcal{V} V}$ and we chose a special one.)

The dual notion, squared bialgebras, is defined as a squared algebra structure plus a coalgebra structure in $\widehat{\mathcal{\otimes} V}$ with compatibility axioms. Unlike the case of vector spaces the notion of squared bicoalgebra is not self-dual, so it has to be distinguished from squared bialgebras. The choice of terminology is motivated by our primary interest in 
comodules rather than in modules. We shall simplify it further dropping the adjective squared and keeping the term bicoalgebra.

Notice that a braiding in $\mathcal{V}$ is not required for work with bicoalgebras. However, if $\mathcal{V}$ is braided any bicoalgebra $B$ generates a braided bialgebra $\circledast B$ in $\mathcal{V}$. Not every braided bialgebra comes from a bicoalgebra in that way.

To introduce Hopf algebras we require that the second dual $X^{\vee \vee}$ of an object $X \in \mathcal{V}$ were isomorphic to $X$ via a functorial isomorphism $\zeta=\zeta_{X}: X \rightarrow X^{\vee \vee}$. Obviously, this condition is weaker than existence of braiding. Given a squared coalgebra $C$ in such $\mathcal{V}$, one can define the opposite coalgebra $C_{\mathrm{op}}$. A (squared) Hopf coalgebra is defined as a bicoalgebra $H$ together with an isomorphism $\gamma: H_{\mathrm{op}} \rightarrow H \in \widehat{\mathcal{\otimes} \mathcal{V}}$ - the antipode - satisfying two equations in $\widehat{\mathcal{\otimes} \mathcal{V}}$. The reason for introducing Hopf coalgebras is the following: the category of comodules over a Hopf coalgebra is rigid and the rigid version of the reconstruction theorem holds: the category of monoidal $\mathbb{k}$-linear exact faithful functors $\omega: \mathcal{C} \rightarrow \mathcal{V}$, where $\mathcal{C}$ is rigid monoidal (and essentially small), and the category of Hopf coalgebras in $V$ are equivalent. The dual notion, squared Hopf algebras, is not equivalent to the notion of Hopf coalgebras.

If, in addition, $\mathcal{V}$ is braided, we get the equivalence of the category of monoidal $\mathbb{k}$ linear exact faithful functors $\omega: \mathcal{C} \rightarrow \mathcal{V}$, where $\mathcal{C}$ is rigid braided, and of the category of quasitriangular Hopf coalgebras, appropriately defined. In particular, the category of comodules over a quasitriangular Hopf coalgebra is braided. This is not trivial, and allows to introduce a non-obvious braiding for the bigger category of comodules over the braided Hopf algebra $\circledast H$. However, it seems impossible, in general, to introduce a braided structure of any kind for the whole category of comodules over a braided Hopf algebra not related with Hopf coalgebras. Thus the notion of a quasitriangular Hopf coalgebra is the closest to the idea of a "quantum group in a braided category".

In particular, applying the (re)construction theorem to the identity functor Id : $\mathcal{V} \rightarrow$ $\mathcal{V}$, we get a quasitriangular Hopf coalgebra structure of the coend

$$
C=\int^{X \in \mathcal{V}} X \odot X^{\vee},
$$

and this is the most interesting case for us. Similar notions exist for ribbon categories.

\section{Tools}

1.1. Tensor product of abelian categories. In this work $\mathbb{k}$ will denote a perfect field.

1.1.1. Definition. We say that an abelian $\mathbb{k}$-linear category $\mathcal{A}$ is a category with length if

1. for any pair of objects $X, Y \in \mathcal{A}$ the $\mathbb{k}$-vector space $\operatorname{Hom}_{\mathcal{A}}(X, Y)$ is finite dimensional, and

2. any object $X \in \mathcal{A}$ has finite length.

The most impressive result from the theory of such categories is:

1.1.2. Proposition (Deligne [1] Corollary 2.17). Let $\mathcal{A}$ be an abelian $\mathbb{k}$-linear category with length. Assume that there exists an object $X \in \mathcal{A}$ such that any object $Y \in \mathcal{A}$ is a 
subquotient of $X^{n}=X \oplus \ldots \oplus X$ for some $n$. Then the category $\mathcal{A}$ is equivalent to the category mod- $A$ for some finite dimensional $\mathbb{k}$-algebra $A$.

From now on $\mathbb{k}$ is a perfect field. We shall remind the definition of a tensor product of abelian $\mathbb{k}$-linear categories, belonging to Deligne [1], in a modified form, using his results, valid in assumption of perfectness.

1.1.3. Definition (following Deligne [1] Definition 5.1). Let $\mathcal{A}_{1}, \ldots, \mathcal{A}_{n}$ be $\mathbb{k}$-linear abelian categories with length. A $\mathbb{k}$-linear abelian category $\mathcal{A}_{1} \otimes \ldots \otimes \mathcal{A}_{n}$, equipped with a $\mathbb{k}$-multilinear, exact in each variable functor

$$
\odot: \mathcal{A}_{1} \times \ldots \times \mathcal{A}_{n} \rightarrow \mathcal{A}_{1} \otimes \ldots \otimes \mathcal{A}_{n}
$$

is called a tensor product of $\mathcal{A}_{1}, \ldots, \mathcal{A}_{n}$ if for each $\mathbb{k}$-linear abelian category $\mathcal{A}$ the induced functor

$$
\underline{\operatorname{Hom}}_{\mathbb{k}, \text { r.e. }}\left(\mathcal{A}_{1} \otimes \ldots \otimes \mathcal{A}_{n}, \mathcal{A}\right) \rightarrow \underline{\operatorname{Hom}}_{\mathbb{k}, \text { r.e. }}\left(\mathcal{A}_{1} \times \ldots \times \mathcal{A}_{n}, \mathcal{A}\right), F \mapsto F \circ \odot
$$

from the category of $\mathbb{k}$-linear right exact functors to the category of $\mathbb{k}$-multilinear right exact in each variable functors is an equivalence.

1.1.4. R e m a r k. Equivalently, one can use left exact functors in the above definition (Deligne [1, Proposition 5.13]).

1.1.5. Proposition (Deligne [1] Proposition 5.13). The tensor product of $\mathbb{k}$-linear abelian categories with length exists and is a category with length. It is unique up to an equivalence. The functor similar to that of Definition 1.1.3

$$
\underline{\operatorname{Hom}}_{\mathbb{k}, \mathrm{e} .}\left(\mathcal{A}_{1} \otimes \ldots \otimes \mathcal{A}_{n}, \mathcal{A}\right) \rightarrow \underline{\operatorname{Hom}}_{\mathbb{k}, \mathrm{e} .}\left(\mathcal{A}_{1} \times \ldots \times \mathcal{A}_{n}, \mathcal{A}\right), F \mapsto F \circ \odot
$$

with the right exact functors replaced by exact functors is also an equivalence. The natural map

$$
\otimes_{i} \operatorname{Hom}\left(X_{i}, Y_{i}\right) \rightarrow \operatorname{Hom}\left(\odot_{i} X_{i}, \odot_{i} Y_{i}\right)
$$

is an isomorphism.

This follows mainly from:

1.1.6. Proposition (Deligne [1] Proposition 5.3, Corollary 5.4). Let $\mathcal{A}_{i}$ be $A_{i}$-mod, where $A_{i}$ are finite dimensional $\mathbb{k}$-algebras, $1 \leqslant i \leqslant n$. Then $\mathcal{A}=A_{1} \otimes_{\mathbb{k}} \ldots \otimes_{\mathbb{k}} A_{n}-\bmod$ equipped with the exterior tensor product functor $\odot:\left(X_{1}, \ldots, X_{n}\right) \mapsto X_{1} \otimes_{\mathbb{k}} \ldots \otimes_{\mathbb{k}} X_{n}$ is a tensor product of $\mathcal{A}_{1}, \ldots, \mathcal{A}_{n}$.

Notice that any category with length $\mathcal{A}$ is a filtered inductive limit of its full subcategories of the form $\langle X\rangle$ - the full subcategory formed by the objects $Y \in \mathcal{A}$, which are subquotients of $X^{n}$ for some $n$. Here $X$ is an object of $\mathcal{A}$. This remark is used together with Propositions 1.1.2, 1.1.6.

Let $\mathcal{A}_{1}, \ldots, \mathcal{A}_{n}, \mathcal{B}_{1}, \ldots, \mathcal{B}_{n}$ be $\mathbb{k}$-linear abelian categories with length and let $T_{i}$ : $\mathcal{A}_{i} \rightarrow \mathcal{B}_{i}$ be $\mathbb{k}$-linear left (resp. right) exact functors. By definition, there exists a $\mathbb{k}$-linear left (resp. right) exact functor

$$
T=\otimes_{i} T_{i}: \otimes_{i} \mathcal{A}_{i} \rightarrow \otimes_{i} \mathcal{B}_{i}, \quad T\left(\odot_{i} X_{i}\right)=\odot_{i} T\left(X_{i}\right) .
$$


1.1.7. Proposition (Deligne [1] Proposition 5.14). If $T_{i}$ are exact (resp. exact and faithful, resp. equivalence of $\mathcal{A}_{i}$ with a full subcategory of $\mathcal{B}_{i}$ stable with respect to subquotients), then $T$ has the same property.

1.2. Monoidal categories. We shall remind only few definitions here. For the developed introduction see [5].

1.2.1. Definition. A rigid category $\mathcal{C}$ is a monoidal category in which for every object $X \in \mathcal{C}$ there exist dual objects $X^{\vee},{ }^{\vee} X \in \mathcal{C}$ and morphisms of evaluation and coevaluation

$$
\begin{aligned}
& \text { ev: } X \otimes X^{\vee} \rightarrow I={ }^{X} \bigcup{ }^{X^{\vee}}, \quad \text { ev: }{ }^{\vee} X \otimes X \rightarrow I={ }^{\vee} X \bigcup{ }^{X}, \\
& \text { coev: } I \rightarrow X^{\vee} \otimes X={ }_{X} \vee \bigcap_{X}, \quad \text { coev: } I \rightarrow X \otimes{ }^{\vee} X={ }_{X} \bigcap^{\vee}{ }^{\prime}
\end{aligned}
$$

satisfying standard equations.

In a rigid monoidal category $\mathcal{C}$ there is a pairing

$$
\begin{aligned}
(X \otimes Y) \otimes\left(Y^{\vee} \otimes X^{\vee}\right) \stackrel{\sim}{\longrightarrow}\left(X \otimes\left(Y \otimes Y^{\vee}\right)\right) \otimes X^{\vee} \rightarrow \\
\stackrel{X \otimes \mathrm{ev} \otimes X^{\vee}}{\longrightarrow}(X \otimes I) \otimes X^{\vee} \stackrel{r_{x} \otimes X^{\vee}}{\longrightarrow} X \otimes X^{\vee} \stackrel{\text { ev }}{\longrightarrow} I,
\end{aligned}
$$

which induces an isomorphism $j_{+X, Y}: Y^{\vee} \otimes X^{\vee} \rightarrow(X \otimes Y)^{\vee}$, such that the above pairing coincides with

$$
(X \otimes Y) \otimes\left(Y^{\vee} \otimes X^{\vee}\right) \stackrel{1 \otimes j_{+}}{\longrightarrow}(X \otimes Y) \otimes(X \otimes Y)^{\vee} \stackrel{\mathrm{ev}}{\longrightarrow} I .
$$

The equation

$$
\begin{aligned}
\operatorname{coev}_{X \otimes Y}=\left(I \stackrel{\operatorname{coev}_{Y}}{\longrightarrow} Y^{\vee} \otimes Y\right. & Y^{\vee} \otimes I \otimes Y \stackrel{1 \otimes \operatorname{coev}_{X} \otimes 1}{\longrightarrow} \\
& \left.Y^{\vee} \otimes X^{\vee} \otimes X \otimes Y \stackrel{j_{+} \otimes 1}{\longrightarrow}(X \otimes Y)^{\vee} \otimes(X \otimes Y)\right)
\end{aligned}
$$

also holds. Similarly, there is an isomorphism $j_{-X, Y}:{ }^{\vee} Y \otimes{ }^{\vee} X \rightarrow{ }^{\vee}(X \otimes Y)$.

There are canonical isomorphisms

$$
X \rightarrow{ }^{\vee}\left(X^{\vee}\right), \quad X \rightarrow\left({ }^{\vee} X\right)^{\vee}
$$

To simplify notations we assume the functors $-{ }^{\vee}$ and ${ }^{\vee}$ - inverse to each other (we can always achieve this replacing the category by an equivalent one). We shall denote the iterated duals by $X^{(n \vee)}=X^{\vee \cdots \vee}\left(n\right.$ times) and $X^{(-n \vee)}={ }^{\vee \cdots \vee} X(n$ times $)$ for $n \geqslant 0$.

The graphical notation for the braiding and its inverse is

$$
c=\left(c_{X, Y}: X \otimes Y \rightarrow Y \otimes X\right)=\overbrace{Y}^{X} \underbrace{Y}_{X}, c^{-1}=\underbrace{Y}_{X} .
$$


In a rigid monoidal braided category there are functorial isomorphisms

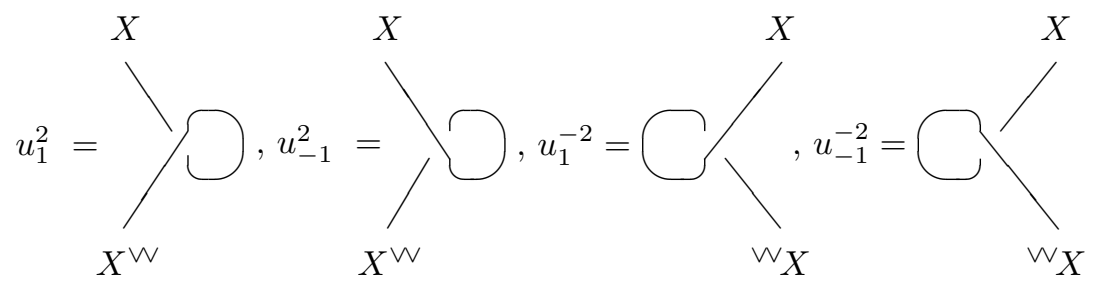

1.3. Monoidal abelian categories. Let $\mathcal{V}=(\mathcal{V}, \otimes, a, I, r, l)$ be a monoidal abelian category. Then it has a canonical $\mathbb{k}$-linear structure, where $\mathbb{k}=\operatorname{End}_{v} I$ is a commutative ring, $I$ is the unit object. For $\lambda \in \operatorname{End}_{\mathcal{V}} I, f \in \operatorname{Hom}_{\mathcal{V}}(X, Y)$ the morphism $\lambda f$ is defined as $\left(X \stackrel{l^{-1}}{\longrightarrow} I \otimes X \stackrel{\lambda \otimes f}{\longrightarrow} I \otimes Y \stackrel{l}{\rightarrow} Y\right)$. We assume that $\mathcal{V}$ is a rigid monoidal abelian category with length and the unit object $I$ such that $\mathbb{k}=$ End $I$ is a perfect field. Then the object $I$ is simple.

The tensor product functor $X \otimes-($ resp. $-\otimes X)$ has a right adjoint $X^{\vee} \otimes-\left(\right.$ resp. $\left.-\otimes^{\vee} X\right)$ and a left adjoint ${ }^{\vee} X \otimes-\left(\right.$ resp. $\left.-\otimes X^{\vee}\right)$. Therefore, the functor $\otimes: \mathcal{V} \times \mathcal{V} \rightarrow \mathcal{V}$ is exact in each variable and it is $\mathbb{k}$-bilinear by the choice of $\mathbb{k}$-linear structure. By Proposition 1.1.5 there exists a $\mathbb{k}$-linear exact functor $\circledast: \mathcal{V} \otimes \mathcal{V} \rightarrow \mathcal{V}$ called the diagonal restriction functor, such that $\otimes$ is isomorphic to the composite

$$
\mathcal{V} \times \mathcal{V} \stackrel{\odot}{\rightarrow} \mathcal{V} \otimes \mathcal{V} \stackrel{\circledast}{\longrightarrow} \mathcal{V} .
$$

The functors $X \mapsto X^{\vee}, X \mapsto{ }^{\vee} X$, quasiinverse to each other, are also exact.

1.3.1. Proposition. The functor $\circledast: \mathcal{V} \otimes \mathcal{V} \rightarrow \mathcal{V}$ is faithful.

1.4. System of notations. The functors $\odot$ and $\circledast$ have their analogues acting between categories $\mathcal{V}^{\otimes n}$ and such. There are several isomorphic functors of that kind. We denote their common value by subindices. So, applying the functor

$$
\mathcal{V}^{\otimes n_{1}} \times \mathcal{V}^{\otimes n_{2}} \times \mathcal{V}^{\otimes n_{3}} \times \ldots \rightarrow \mathcal{V}^{\otimes p}
$$

to $(A, B, C, \ldots)$ we get $A_{i_{1} \ldots i_{n_{1}}} \otimes B_{j_{1} \ldots j_{n_{2}}} \otimes C_{k_{1} \ldots k_{n_{3}}} \otimes \ldots$, where the sign $\otimes$ may be changed to $\odot$ or $\circledast$ for purely aesthetical reasons and contains no additional information. Everything is encoded in terms of indices, which are all distinct and are taken from the set

$$
\begin{aligned}
& \left\{i_{1}, \ldots, i_{n_{1}}, j_{1}, \ldots, j_{n_{2}}, k_{1}, \ldots, k_{n_{3}}, \ldots\right\} \\
& \quad=\left\{1^{\prime}, 1^{\prime \prime}, 1^{\prime \prime \prime}, \ldots, 1^{m_{1}}, 2^{\prime}, 2^{\prime \prime}, 2^{\prime \prime \prime}, \ldots, 2^{m_{2}}, 3^{\prime}, \ldots, 3^{m_{3}}, \ldots, p^{\prime}, \ldots, p^{m_{p}}\right\} .
\end{aligned}
$$

The number means the tensorand in $\mathcal{V} \otimes p$ to which the present tensorand goes, and the superscripts determine the order in which several terms are tensored to get an object of $\mathcal{V}$ - tensorand from $\mathcal{V}^{\otimes p}$. Another way to describe such functor is to give a permutation from $\mathfrak{S}_{n}$ and two partitions $n=n_{1}+n_{2}+n_{3}+\ldots=m_{1}+m_{2}+\ldots+m_{p}$.

Examples. If $X, Y \in \mathcal{V}, C \in \mathcal{V} \otimes \mathcal{V}$ then $X_{1^{\prime}} \otimes Y_{1^{\prime \prime}}$ denotes the usual tensor product of $X$ and $Y, X_{1^{\prime \prime}} \otimes Y_{1^{\prime}}$ is $Y \otimes X$. Similarly, $C_{1^{\prime} 1^{\prime \prime}}$ is $\circledast C$ and $C_{1^{\prime \prime} 1^{\prime}}$ is $\circledast P C$, where $P: \mathcal{V} \otimes \mathcal{V} \rightarrow \mathcal{V} \otimes \mathcal{V}$ is the permutation functor, $P(X \odot Y)=Y \odot X$. The three objects $X_{1} \odot C_{23}, X_{2} \odot C_{13}, C_{12} \odot X_{3}$ of $\mathcal{V} \otimes 3$ differ by the place where $X$ goes. Applying $\circledast \otimes \operatorname{Id}_{\mathcal{V}}$ : 
$\mathcal{V}^{\otimes 3} \rightarrow \mathcal{V}^{\otimes 2}$ to $X_{1} \odot C_{23}$ we get $X_{1^{\prime}} \otimes C_{1^{\prime \prime} 2}$. Applying $\operatorname{Id}_{\mathcal{V}} \otimes \circledast: \mathcal{V}^{\otimes 3} \rightarrow \mathcal{V}^{\otimes 2}$ to $C_{12} \odot X_{3}$ we get $C_{12^{\prime}} \otimes X_{2^{\prime \prime}}$. The functor $\operatorname{Id}_{\mathcal{V}} \otimes \circledast \otimes \operatorname{Id}_{\mathcal{V}}: \mathcal{V} \otimes 4 \rightarrow \mathcal{V} \otimes 3$ (tensoring the second and the third places), applied to $C \odot C$, gives $C_{12^{\prime}} \otimes C_{2^{\prime \prime} 3}$.

To use the same system of notations for morphisms we should write indices for both source and target. However, we shall abbreviate the notation using only one set of indices, either for the source, or for the target. Thus, instead of

$$
\begin{gathered}
{ }_{1^{\prime} 1^{\prime \prime}} \varepsilon_{1}: C_{1^{\prime} 1^{\prime \prime}} \rightarrow I_{1}, \\
{ }_{12^{\prime}} C_{12^{\prime}} \otimes{ }_{2^{\prime \prime} 2^{\prime \prime \prime}} \varepsilon_{2^{\prime \prime}}: C_{12^{\prime}} \otimes C_{2^{\prime \prime} 2^{\prime \prime \prime}} \rightarrow C_{12^{\prime}} \otimes I_{2^{\prime \prime}}
\end{gathered}
$$

we write $\varepsilon: C_{1^{\prime} 1^{\prime \prime}} \rightarrow I$ and $C_{12^{\prime}} \otimes \varepsilon_{2^{\prime \prime}}$. Instead of

$$
\begin{gathered}
{ }_{123} \Delta_{12^{\prime} 2^{\prime \prime} 3}: C_{13} \odot I_{2} \rightarrow C_{12^{\prime}} \otimes C_{2^{\prime \prime} 3}, \\
{ }_{124} \Delta_{12^{\prime} 2^{\prime \prime} 4} \odot I_{3}: C_{14} \odot I_{2} \odot I_{3} \rightarrow C_{12^{\prime}} \otimes C_{2^{\prime \prime} 4} \odot I_{3}
\end{gathered}
$$

we write $\Delta_{123}$ and $\Delta_{124} \odot I_{3}$

Sometimes we simplify $X_{1^{\prime}}, X_{1^{\prime \prime}}, X_{1^{\prime \prime \prime}}, X_{1^{4}}, \ldots$ to $X^{1}, X^{2}, X^{3}, X^{4}, \ldots$

1.5. Monoidal structures on $\mathcal{V} \otimes \mathcal{V}$. Let $\mathcal{V}$ be a $\mathbb{k}$-linear abelian monoidal category with length. Then $\mathcal{V} \otimes \mathcal{V}$ has a monoidal structure as well. In fact, there are four monoidal structures, since we may choose between $(\mathcal{V}, \otimes)$ and $\left(\mathcal{V}, \otimes_{\mathrm{op}}\right)$ in both tensorands. The main monoidal structure, which we fix from now on, is:

$$
\begin{aligned}
\bar{\otimes}:(\mathcal{V} \otimes \mathcal{V}) \times(\mathcal{V} \otimes \mathcal{V}) & \longrightarrow \mathcal{V} \otimes \mathcal{V} \\
\left(A_{12}, B_{12}\right) & \longmapsto A_{1^{\prime} 2^{\prime \prime} \otimes B_{1^{\prime \prime} 2^{\prime}}} \\
(X \odot Y, V \odot W) & \longmapsto(X \otimes V) \odot(W \otimes Y) .
\end{aligned}
$$

1.5.1. Theorem. If $(\mathcal{V}, \otimes)$ is rigid, then $(\mathcal{V} \otimes \mathcal{V}, \bar{\otimes})$ is rigid as well.

1.6. Ind-objects. Following Grothendieck and Verdier [3] we consider the category of ind-objects of a given $\mathbb{k}$-linear abelian category $\mathcal{A}$. Recall that an ind-object of $\mathcal{A}$ is a functor $X: I \rightarrow \mathcal{A}$ from a filtered partially ordered set $I$, in particular, an arrow $x_{i j}: X_{i} \rightarrow X_{j} \in \mathcal{A}$ is given for $i<j, i, j \in I$. The set of morphisms from $X: I \rightarrow \mathcal{A}$ to $Y: J \rightarrow \mathcal{A}$ is

$$
\underset{I}{\lim _{I}} \underset{J}{\lim } \operatorname{Hom}\left(X_{i}, Y_{j}\right) .
$$

We denote the category of ind-objects of $\mathcal{A}$ by $\widehat{\mathcal{A}}$ as a synonym of standard notation $\operatorname{Ind}(\mathcal{A})$. The category $\widehat{\mathcal{A}}$ is a $\mathbb{k}$-linear abelian category [3, Exercise 8.9.9]. Small projective and inductive limits in $\widehat{\mathcal{A}}$ are representable in $\widehat{\mathcal{A}}$ (Grothendieck and Verdier [3, Propositions 8.9.1 and 8.9.5]).

1.6.1. Theorem (Grothendieck and Verdier [3] Theorem 8.3.3).Let $\mathcal{A}$ be essentially small, then the functor

$$
\begin{aligned}
& \widehat{\mathcal{A}} \longrightarrow \underline{\operatorname{Hom}}_{\mathbb{k}, \text { l.e. }}\left(\mathcal{A}^{\text {op }}, \mathbb{k} \text {-Vect }\right), \\
& (X: I \rightarrow \mathcal{A}) \longmapsto\left(Y \mapsto \underset{I}{\lim } \operatorname{Hom}\left(Y, X_{i}\right)\right)
\end{aligned}
$$

with values in the category of $\mathbb{k}$-linear left exact functors is an equivalence of categories. 
If $\mathcal{A}, \mathcal{B}$ are $\mathbb{k}$-linear abelian essentially small categories, then any functor $F: \mathcal{A} \rightarrow \mathcal{B}$ extends to a functor $\hat{F}: \widehat{\mathcal{A}} \rightarrow \widehat{\mathcal{B}}, X \mapsto F \circ X$. If $F$ is $\mathbb{k}$-linear (resp. right exact, resp. exact), so is $\hat{F}$ (Grothendieck and Verdier [3, Corollary 8.9.8]). The functor $\hat{F}: \widehat{\mathcal{A}} \rightarrow \widehat{\mathcal{B}}$ commutes with filtered inductive limits [3, Proposition 8.6.3]. If $F$ is right exact, $\hat{F}$ commutes with arbitrary inductive limits.

1.6.2. Proposition. Let any object of $\mathcal{A}$ have finite length. Then the category $\widehat{\mathcal{A}}$ is equivalent to its full subcategory consisting of functors $X: I \rightarrow \mathcal{A}$ such that $x_{i j}: X_{i} \rightarrow X_{j}$ is a monomorphism for any pair $i<j$.

1.6.3. Remark. Let $X: I \rightarrow \mathcal{A}$ be in $\widehat{\mathcal{A}}$ and let $J \subset I$ be a cofinal set. Then the ind-object $X^{\prime}=\left.X\right|_{J}: J \rightarrow \mathcal{A}$ is isomorphic to $X$ in $\widehat{\mathcal{A}}$.

Let $X: I \rightarrow \mathcal{A}$ be an ind-object such that $x_{i j}: X_{i} \rightarrow X_{j}$ are monomorphisms, $i<j$. We say that $X$ is closed under intersections if

(a) for any subset $J \subset I$ there is an element $i=\cap J \in I$ such that $i \leqslant j$ for any $j \in J$ and $i$ is the biggest element with this property;

(b) for any subset $J \subset I$ there is a finite subset $J^{\prime} \subset J$ such that for any finite $K$, $J^{\prime} \subset K \subset J$, and any $r \geqslant K$ the subobject $X_{i}$ is the intersection of subobjects $X_{k}$, $k \in K$, in $X_{r}$, that is, the canonical morphism

$$
X_{i} \rightarrow \lim _{k \in K}\left(X_{k} \stackrel{x_{k r}}{\longrightarrow} X_{r}\right)
$$

is an isomorphism.

1.6.4. Proposition. (a) Any ind-object is isomorphic in $\widehat{\mathcal{A}}$ to an ind-object closed under intersections.

(b) Let $X: I \rightarrow \mathcal{A}, Y: J \rightarrow \mathcal{A}$ be ind-objects. Assume that $Y$ is closed under intersections. Then any morphism $f: X \rightarrow Y \in \widehat{\mathcal{A}}$ can be represented by a monotonous map $m: I \rightarrow J$ and a family of morphisms $f_{i}: X_{i} \rightarrow Y_{m(i)}$.

\section{Squared coalgebras}

2.1. Definitions. Let $\mathcal{V}$ be a $\mathbb{k}$-linear abelian rigid monoidal category with length.

2.1.1. Definition. A squared coalgebra $C=(C, \Delta, \varepsilon)$ in $\widehat{\mathcal{V}}$ is an object $C \in \widehat{\mathcal{\otimes} V}$ equipped with a comultiplication $\Delta_{123}: C_{13} \odot I_{2} \rightarrow C_{12^{\prime}} \otimes C_{2^{\prime \prime} 3} \in \widehat{\mathcal{V} \otimes 3^{3}}$ and a counit $\varepsilon: C_{1^{\prime} 1^{\prime \prime}} \rightarrow I \in \widehat{\mathcal{V}}$, such that coassociativity holds:

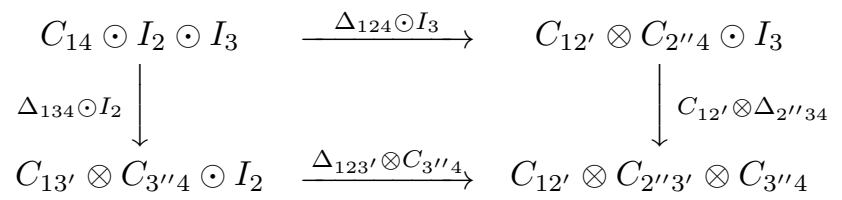

and $\varepsilon$ is the counit:

$$
\begin{aligned}
& \left(C_{12} \stackrel{\sim}{\longrightarrow} C_{1^{\prime} 2} \otimes I_{1^{\prime \prime}} \stackrel{\Delta_{1^{\prime} 1^{\prime \prime} 2}^{\longrightarrow}}{\longrightarrow} C_{1^{\prime} 1^{\prime \prime}} \otimes C_{1^{\prime \prime \prime} 2} \stackrel{\varepsilon \otimes C}{\longrightarrow} I_{1^{\prime}} \otimes C_{1^{\prime \prime} 2} \stackrel{\sim}{\longrightarrow} C_{12}\right)=\operatorname{id}_{C} \\
& \left(C_{12} \stackrel{\sim}{\longrightarrow} I_{2^{\prime}} \otimes C_{12^{\prime \prime}} \stackrel{\Delta_{12^{\prime} 2^{\prime \prime}}}{\longrightarrow} C_{12^{\prime}} \otimes C_{2^{\prime \prime} 2^{\prime \prime \prime}} \stackrel{C \otimes \varepsilon}{\longrightarrow} C_{12^{\prime}} \otimes I_{2^{\prime \prime}} \stackrel{\sim}{\longrightarrow} C_{12}\right)=\operatorname{id}_{C}
\end{aligned}
$$


Graphical notations partially explain the choice of indices and help to memorise them. Comultiplication is denoted

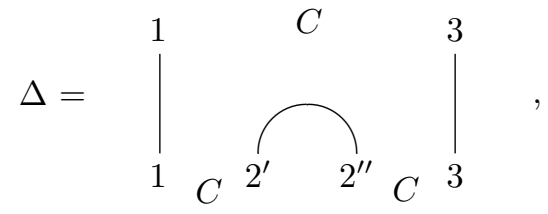

the counit is denoted

$$
\varepsilon=1^{\prime} C 1^{\prime \prime}
$$

The coassociativity equation is

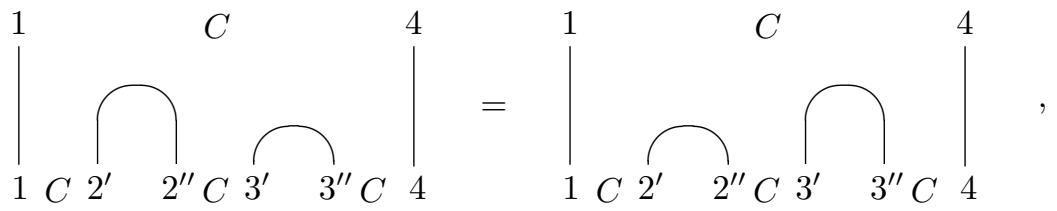

the equations for the counit are

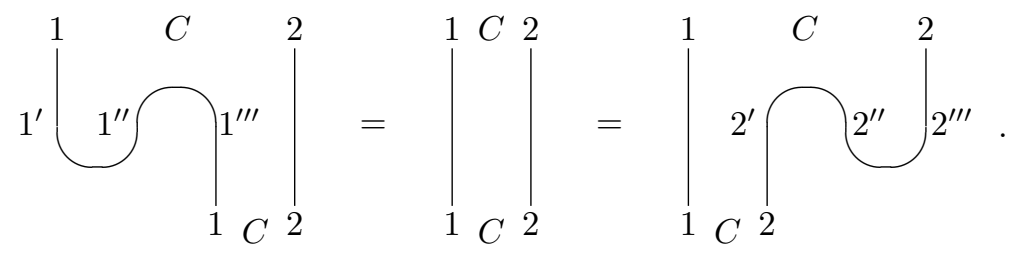

2.1.2. Definition. A squared coalgebra homomorphism $\left(C, \Delta, \varepsilon_{C}\right) \rightarrow\left(D, \Delta, \varepsilon_{D}\right)$ is a morphism $f: C \rightarrow D \in$ Mor $\widehat{\mathcal{V} \otimes \mathcal{V}}$ such that

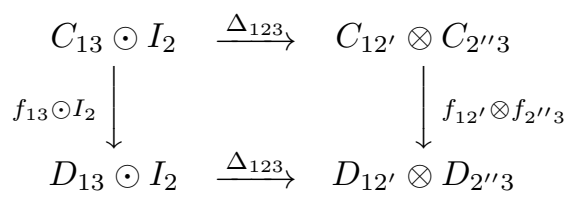

commutes and equation

$$
\left(\circledast C \stackrel{\circledast f}{\longrightarrow} \circledast D \stackrel{\varepsilon_{D}}{\longrightarrow} I\right)=\varepsilon_{C}
$$

holds.

Squared coalgebras form a category denoted Coalgsq $(\widehat{\mathcal{V}})$. Its full subcategory consisting of squared coalgebras, which are objects of $\mathcal{V} \otimes \mathcal{V}$, is denoted $\operatorname{Coalgsq}(\mathcal{V})$.

\subsection{Comodules}

2.2.1. Definition. A left comodule $X \in \widehat{\mathcal{V}}$ over a squared coalgebra $C$ is an object $X$ of $\widehat{v}$ equipped with the coaction

$$
\delta=\delta_{X}: X_{1} \odot I_{2} \rightarrow C_{12^{\prime}} \otimes X_{2^{\prime \prime}} \in \widehat{\mathcal{V} \otimes \mathcal{V}}
$$


which is coassociative:

and counital:

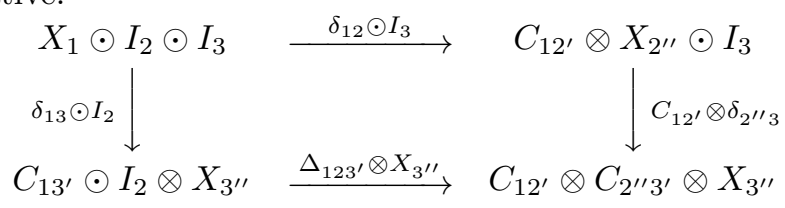

$$
(X \stackrel{\sim}{\longrightarrow} X \otimes I \stackrel{\circledast \delta}{\longrightarrow}(\circledast C) \otimes X \stackrel{\varepsilon \otimes X}{\longrightarrow} I \otimes X \stackrel{\sim}{\longrightarrow} X)=\operatorname{id}_{X} .
$$

Graphical notation for the coaction is

$$
\delta=\left.\right|_{1} ^{1} X
$$

and it will be explained later. Coassociativity takes the form

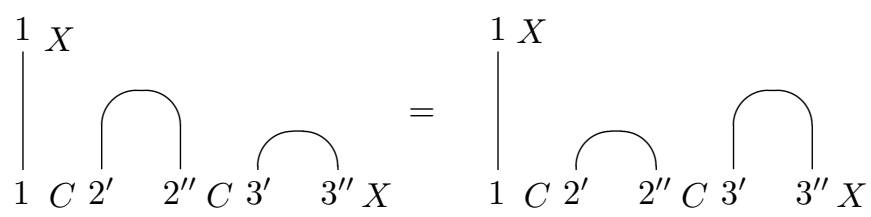

and counitality is

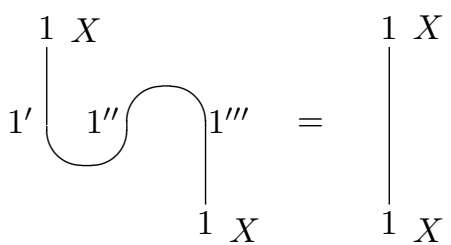

The definition above should be generalised. Let $\mathcal{A}$ be a $\mathbb{k}$-linear abelian category with length.

2.2.2. Definition. A left comodule $X \in \widehat{V \otimes \mathcal{A}}$ over a squared coalgebra $C \in$ $\operatorname{Coalgsq}(\widehat{\mathcal{V}})$ is an object $X=X_{10} \in \widehat{\mathcal{V}} \mathcal{A}$ equipped with the coaction

$$
\delta=\delta_{X}: X_{10} \odot I_{2} \rightarrow C_{12^{\prime}} \otimes X_{2^{\prime \prime} 0} \in(\mathcal{V} \otimes \mathcal{V} \otimes \mathcal{A})^{\wedge}
$$

such that coassociativity $(2.2 .1)$ in $\left(\mathcal{V}^{\otimes 3} \otimes \mathcal{A}\right)^{\wedge}$ and counitality $(2.2 .2)$ in $\widehat{\mathcal{\otimes} A}$ hold.

When $\mathcal{A}=\mathbb{k}$-vect this reduces to the previous definition.

2.2.3. Definition. A morphism of left $C$-comodules $\left(X, \delta_{X}\right) \rightarrow\left(Y, \delta_{Y}\right)$ in $\widehat{\mathcal{V}}$ (resp. in $\widehat{\mathcal{\otimes}} \mathcal{A}$ ) is $f: X \rightarrow Y \in \widehat{\mathcal{V}}$ (resp. $f: X_{10} \rightarrow Y_{10} \in \widehat{\mathcal{V} \otimes \mathcal{A}}$ ) such that

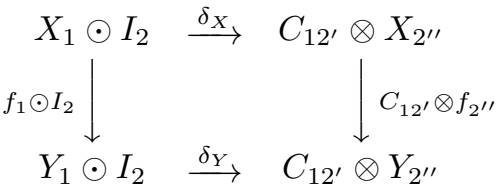

(resp. the same diagram in $\left.(\mathcal{V} \otimes \mathcal{V} \otimes \mathcal{A})^{\wedge}\right)$ commutes. 
Left $C$-comodules form a category, which is denoted ${ }^{C} \widehat{\mathcal{V}}$ (resp. ${ }^{C} \widehat{\mathcal{\otimes}} \mathcal{A}$ ). It has a full subcategory ${ }^{C} \mathcal{V}$ (resp. ${ }^{C}(\mathcal{V} \otimes \mathcal{A})$ ) formed by objects from $\mathcal{V}($ resp. $\mathcal{V} \otimes \mathcal{A})$.

It is easy to show that if $f: X \rightarrow Y$ is a morphism of comodules in ${ }^{C} \widehat{\mathcal{V}}$ (resp. ${ }^{C} \widehat{\mathcal{\otimes}} \mathcal{A}$, $\left.{ }^{C} \mathcal{V},{ }^{C}(\mathcal{V} \otimes \mathcal{A})\right)$, and

$$
\text { Ker } f \stackrel{\operatorname{ker} f}{\longrightarrow} X \stackrel{\operatorname{coim} f}{\longrightarrow} \operatorname{Coim} f \simeq \operatorname{Im} f \stackrel{\operatorname{im} f}{\longrightarrow} Y \stackrel{\operatorname{coker} f}{\longrightarrow} \text { Coker } f
$$

is its canonical decomposition in $\widehat{\mathcal{V}}$ (resp. $\widehat{\mathcal{\otimes}} \mathcal{A}, \mathcal{V}, \mathcal{V} \otimes \mathcal{A}$ ), then the objects Ker $f$, $\operatorname{Coim} f, \operatorname{Im} f$, Coker $f$ have unique $C$-comodule structure such that the morphisms above are morphisms of comodules. It follows:

2.2.4. Proposition. The category ${ }^{C} \widehat{\mathcal{V}}\left(\right.$ resp. $\left.{ }^{C} \widehat{\mathcal{V} \otimes} \mathcal{A},{ }^{C} \mathcal{V},{ }^{C}(\mathcal{V} \otimes \mathcal{A})\right)$ is $\mathbb{k}$-linear and

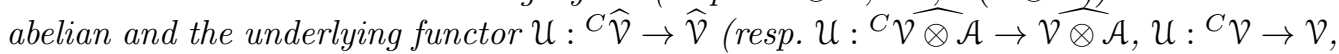
$\left.\mathcal{U}:{ }^{C}(\mathcal{V} \otimes \mathcal{A}) \rightarrow \mathcal{V} \otimes \mathcal{A}\right)$ is exact and faithful.

Any $\mathbb{k}$-linear exact functor $F: \mathcal{A} \rightarrow \mathcal{B}$ induces a $\mathbb{k}$-linear exact functor $\mathcal{V} \otimes F$ : $C \widehat{\mathcal{A} A} \rightarrow C \widehat{\mathcal{Q} \otimes \mathcal{B}}$.

2.2.5. Example. $(C, \Delta)$ is a $C$-comodule from ${ }^{C} \widehat{\mathcal{\otimes} V}$ (resp. $\widehat{\mathcal{\otimes} V \mathcal{V}}{ }^{C}$ ). It is called the left regular comodule.

\subsection{The fundamental theorem on coalgebras}

2.3.1. ThEOREM. Any comodule from ${ }^{C} \widehat{\mathcal{V}}$ (resp. ${ }^{C} \widehat{\mathcal{\otimes} A}$ ) is a union, i.e. filtered inductive limit, of its subcomodules from ${ }^{C} \mathcal{V}\left(\right.$ resp. $\left.{ }^{C}(\mathcal{V} \otimes \mathcal{A})\right)$.

2.3.2. Corollary. $\widehat{C \mathcal{V}} \simeq C \widehat{\mathcal{V}}$ and $\left({ }^{C}(\mathcal{V} \otimes \mathcal{A})\right)^{\wedge} \simeq{ }^{C} \widehat{\mathcal{\otimes}} \mathcal{A}$.

2.3.3. TheOREM (fundamental theorem on coalgebras). A squared coalgebra $C \in$ Coalgsq $(\widehat{\mathcal{V}})$ is a filtered inductive limit of its subcoalgebras from $\operatorname{Coalgsq}(\mathcal{V})$.

Let $\mathcal{A}$ be a $\mathbb{k}$-linear abelian category with length and let $C$ be a squared coalgebra in $\mathcal{V}$.

2.3.4. TheOrem. The functor $\Psi:\left({ }^{C} \mathcal{V}\right) \otimes \mathcal{A} \rightarrow{ }^{C}(\mathcal{V} \otimes \mathcal{A})$ induced by $\left({ }^{C} \mathcal{V}\right) \times \mathcal{A} \rightarrow$ $C^{C}(\mathcal{V} \otimes \mathcal{A}),(X, M) \mapsto X \odot M$, is an equivalence.

2.4. The category of fibre functors. Let $\mathcal{V}$ be a $\mathbb{k}$-linear abelian category. Extending the definition of Saavedra [7] let us call a fibre functor to $\mathcal{V}$ a $\mathbb{k}$-linear exact faithful functor $a: \mathcal{A} \rightarrow \mathcal{V}$, where $\mathcal{A}$ is a $\mathbb{k}$-linear abelian essentially small category. Following Schauenburg [8] we define the category of fibre functors.

2.4.1. Definition. Let the category $\mathfrak{A}=\mathfrak{A}(\mathcal{V})$ have fibre functors to $\mathcal{V}$ as objects and let morphisms from $a: \mathcal{A} \rightarrow \mathcal{V}$ to $b: \mathcal{B} \rightarrow \mathcal{V}$ be equivalence classes of pairs $(F, \phi)$, where $F: \mathcal{A} \rightarrow \mathcal{B}$ is a functor and $\phi: a \stackrel{\sim}{\longrightarrow} b F$ is a functorial isomorphism. Two such pairs $(F, \phi)$ and $(G, \gamma)$ are equivalent if there is a functorial isomorphism $\zeta: F \rightarrow G$ such that

$$
\gamma=(a \stackrel{\phi}{\rightarrow} b F \stackrel{b \zeta}{\rightarrow} b G)
$$

The composite of two morphisms represented by $(F, \phi)$ and $(G, \gamma)$ is represented by $(G F, \gamma F \circ \phi)$. Clearly, $\operatorname{Hom}_{\mathfrak{A}}(a, b)$ is a set. 
Now let $\mathcal{V}$ be a $\mathbb{k}$-linear abelian rigid monoidal category with length.

There is a functor $\Phi: \operatorname{Coalgsq}(\widehat{\mathcal{V}}) \rightarrow \mathfrak{A}(\mathcal{V}), C \mapsto\left(\mathcal{U}:{ }^{C} \mathcal{V} \rightarrow \mathcal{V}\right)$, where $\mathcal{U}$ is the underlying functor. To a squared coalgebra morphism $f: C \rightarrow D$ corresponds the equivalence class of the pair $(F, \phi)$, where

$$
F\left(X, \delta_{X}\right)=\left(X, X_{1} \odot I_{2} \stackrel{\delta_{X}}{\longrightarrow} C_{12^{\prime}} \otimes X_{2^{\prime \prime}} \stackrel{f \otimes X}{\longrightarrow} D_{12^{\prime}} \otimes X_{2^{\prime \prime}}\right)
$$

and $\phi$ is the identity automorphism $\mathcal{U} \rightarrow \mathcal{U}$.

Our primary goal is to show that the functor $\Phi$ is an equivalence.

2.4.2. The coend Let $\mathcal{C}$ be a $\mathbb{k}$-linear abelian category with length. The coend $C$ of a bifunctor $B: \mathcal{P} \times \mathcal{P}^{\circ} \rightarrow \mathcal{C}$ is defined in [5] as an object of $\widehat{\mathcal{C}}$ which is the inductive limit of the diagram

$$
B(X, X) \stackrel{B(X, f)}{\longleftarrow} B(X, Y) \stackrel{B(f, Y)}{\longrightarrow} B(Y, Y),
$$

where $f: X \rightarrow Y$ runs over Mor $\mathcal{P}$. That is, $C$ is equipped with a morphism $i_{X}$ : $B(X, X) \rightarrow C \in \widehat{\mathcal{C}}$ for each $X \in \mathrm{Ob} \mathcal{P}$, the diagram

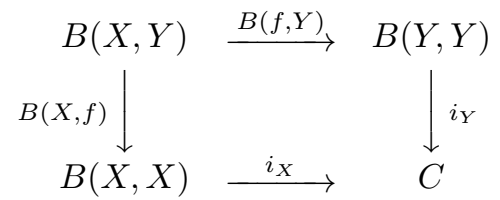

is commutative for any $f: X \rightarrow Y \in \operatorname{Mor} \mathcal{P}$, and $C$ is universal between such objects. If $\mathcal{P}$ is small, we can say that the sequence

$$
\bigoplus_{f: X \rightarrow Y \in \text { Mor } \mathcal{P}} B(X, Y) \stackrel{B(X, f)-B(f, Y)}{\longrightarrow} \bigoplus_{X \in \text { Ob } \mathcal{P}} B(X, X) \stackrel{\oplus i_{X}}{\longrightarrow} C \rightarrow 0
$$

is exact. So in this case the coend exists. More generally, it exists for essentially small $\mathcal{P}$. It is denoted $C=\int^{X \in \mathcal{P}} B(X, X)$.

Let us consider the particular case. Let $p: \mathcal{P} \rightarrow \mathcal{V}$ be a functor from an essentially small category $\mathcal{P}$, let $\mathcal{C}=\mathcal{V} \otimes \mathcal{V}$ and let $B: \mathcal{P} \times \mathcal{P}$ op $\rightarrow \mathcal{C}, B(X, Y)=p X \odot(p Y)^{\vee}$. The coend is denoted

$$
C=\int^{X \in \mathcal{P}} p X \odot(p X)^{\vee} .
$$

The object $M \odot M^{\vee} \in \mathcal{V} \otimes \mathcal{V}$ has a canonical squared coalgebra structure for any $M \in \mathcal{V}$. Namely,

$$
\begin{gathered}
\Delta_{123}=M \odot \operatorname{coev} \odot M^{\vee}: M \odot I \odot M^{\vee} \rightarrow M \odot M^{\vee} \otimes M \odot M^{\vee}, \\
\varepsilon=\mathrm{ev}: M \otimes M^{\vee} \rightarrow I .
\end{gathered}
$$

Compare these formulae with the graphical notations on page 120 . The object $M$ has a canonical structure of a left $M \odot M^{\vee}$-comodule, namely,

$$
\delta=M \odot \operatorname{coev}: M_{1} \odot I_{2} \rightarrow M_{1} \odot M_{2^{\prime}}^{\vee} \otimes M_{2^{\prime \prime}} .
$$

Compare with the graphical notations on page 121. In particular, this holds for $M=p X$.

2.4.3. Proposition. If the coend (2.4.2) exists, it has a unique squared coalgebra structure such that the structure morphisms $i_{X}: p X \odot(p X)^{\vee} \rightarrow C$ are coalgebra morphisms. 
Since $p X$ is a $p X \odot(p X)^{\vee}$-comodule, it is a $C$-comodule as well for any $X \in \mathrm{Ob} \mathcal{P}$.

2.4.4. Proposition. If the coend $C$ exists, the map $\mathrm{Ob} \mathcal{P} \rightarrow \mathrm{Ob}^{C} \mathcal{V}, X \mapsto(p X, \delta)$ extends to the functor $F: \mathcal{P} \rightarrow^{C} \mathcal{V}, f \mapsto p f$ such that $p=\left(\mathcal{P} F_{\longrightarrow} C \mathcal{V} \stackrel{u}{\longrightarrow} \mathcal{V}\right)$.

2.5. Reconstruction theorems. Now we come back to the case of a fibre functor $a$ : $\mathcal{A} \rightarrow \mathcal{V}$. Assume that $\mathcal{P} \subset \mathcal{A}$ is a full subcategory equivalent to $\mathcal{A}$ and $\mathcal{O}=\mathrm{Ob} \mathcal{P}$ is a set. Denote $p=\left.a\right|_{\mathcal{P}}: \mathcal{P} \rightarrow \mathcal{V}$. Notice, that coend (2.4.2) serves as the coend of the bifunctor $\mathcal{A}^{\mathrm{op}} \times \mathcal{A} \rightarrow \mathcal{V} \otimes \mathcal{V},(X, Y) \mapsto a X \odot(a Y)^{\vee}$ as well.

2.5.1. TheOREM. The functor $F: \mathcal{A} \rightarrow{ }^{C} \mathcal{V}$ is an equivalence of categories.

In particular case $\mathcal{V}=\mathbb{k}$-vect this theorem was proved by Saavedra [7] (see also Schauenburg [8]).

2.5.2. Theorem. (a) The map $(a: \mathcal{A} \rightarrow \mathcal{V}) \longmapsto C_{a}$ constructed in Section 2.4 .2 extends to a functor

$$
\Psi: \mathfrak{A}(\mathcal{V}) \rightarrow \operatorname{Coalgsq}(\widehat{\mathcal{V}}) .
$$

(b) The functor $F_{a}: \mathcal{A} \rightarrow \Phi(\Psi(\mathcal{A}))={ }^{C_{a}} \mathcal{V}$ constructed in Theorem 2.5 .1 together with id $: a \rightarrow \mathcal{U} \circ F_{a}$ gives an isomorphism of functors

$$
F: \operatorname{Id}_{\mathfrak{A}(\mathcal{V})} \stackrel{\sim}{\longrightarrow} \Phi \Psi .
$$

(c) The functors $\Phi:$ Coalgsq $(\widehat{\mathcal{V}}) \rightarrow \mathfrak{A}(\mathcal{V}), C \mapsto{ }^{C} \mathcal{V}$, and $\Psi: \mathfrak{A}(\mathcal{V}) \rightarrow \operatorname{Coalgsq}(\widehat{\mathcal{V}})$, $a \rightarrow C_{a}$, are equivalences, quasiinverse to each other.

In the case of $\mathcal{V}=\mathbb{k}$-vect this theorem was proved by Schauenburg [8].

Constructing another adjunction $\Psi \Phi \stackrel{\sim}{\longrightarrow} \mathrm{Id}$, is also of practical interest.

2.5.3. Proposition. (a) Let $C$ be a squared coalgebra in $\widehat{\mathcal{V}}$ and let $X \in \mathcal{V}$. The structures of $C$-comodules in $X$ and squared coalgebra homomorphisms $X \odot X^{\vee} \rightarrow C$ are in bijective correspondence.

(b) If $\delta: X_{1} \odot I_{2} \rightarrow C_{12^{\prime}} \otimes X_{2^{\prime \prime}}$ is a comodule structure, then

$$
\begin{aligned}
\ddot{\imath}=\ddot{i}_{X}= & \left(X_{1} \odot X_{2}^{\vee} \stackrel{\sim}{\longrightarrow} X_{1} \odot I_{2^{\prime}} \otimes X_{2^{\prime \prime}}^{\vee} \stackrel{\delta_{12^{\prime}} \otimes X_{2^{\prime \prime}}^{\vee}}{\longrightarrow}\right. \\
& \left.C_{12^{\prime}} \otimes X_{2^{\prime \prime}} \otimes X_{2^{\prime \prime \prime}}^{\vee} \stackrel{C_{12^{\prime}} \otimes \mathrm{ev}_{2^{\prime \prime}}}{\longrightarrow} C_{12^{\prime}} \otimes I_{2^{\prime \prime}} \stackrel{\simeq}{\longrightarrow} C_{12}\right)
\end{aligned}
$$

is a homomorphism of squared coalgebras.

(c) If $\ddot{i}_{X}: X_{1} \odot X_{2}^{\vee} \rightarrow C_{12}$ is a squared coalgebra homomorphism, then

$$
\delta=\left(X_{1} \odot I_{2} \stackrel{X_{1} \odot \operatorname{coev}_{2}}{\longrightarrow} X_{1} \odot X_{2^{\prime}}^{\vee} \otimes X_{2^{\prime \prime}} \stackrel{\ddot{i}_{12^{\prime}} \otimes X_{2^{\prime \prime}}}{\longrightarrow} C_{12^{\prime}} \otimes X_{2^{\prime \prime}}\right)
$$

is a $C$-comodule structure on $X$.

(d) There is a unique squared coalgebra homomorphism $h_{C}: C^{\prime} \stackrel{\text { def }}{=} \Psi(\Phi C) \rightarrow C$ such that for any $X \in{ }^{C} \mathcal{V}$ the composite coalgebra morphism $\ddot{\imath}_{X}: X \odot X^{\vee} \stackrel{i_{X}}{\longrightarrow} C^{\prime} \stackrel{h_{C}}{\longrightarrow} C$ is the canonical (2.5.1).

(e) The family $h_{C}: \Psi(\Phi C) \rightarrow C$ gives an isomorphism of functors $h: \Psi \Phi \rightarrow$ $\operatorname{Id}_{\text {Coalgsq }(\widehat{\mathcal{V}})}$.

2.5.4. Corollary. Any squared coalgebra $C$ is the union of the images of the canonical morphisms (2.5.1) for all comodules $X$. 
This is a detailed form of the fundamental theorem on coalgebras 2.3.3.

2.6. Comodules over ordinary coalgebras. Let $C$ be a squared coalgebra in $\mathcal{V}$. Then $\bar{C}=\circledast C \in \mathcal{V}$ is an ordinary coalgebra in $\mathcal{V}$. How big is the category of $\bar{C}$-comodules in comparison with $C$-comodules?

First of all, any $\bar{C}$-comodule is a union of its rigid subcomodules, $\bar{C} \widehat{\mathcal{V}}=\widehat{\overline{C V}}$. This is a general fact. It follows from the embedding $\delta: X \hookrightarrow \bar{C} \otimes X$ of $\bar{C}$-comodules and the fundamental theorem on coalgebras.

2.6.1. THEOREM. The functor

$$
\begin{gathered}
{ }^{C} \mathcal{V} \times \mathcal{V} \stackrel{\otimes}{\longrightarrow} \mathcal{V}, \quad\left(X, \delta_{X}\right) \times Y \mapsto\left(X \otimes Y, \bar{\delta}_{X} \otimes Y\right) \\
\bar{\delta}_{X}=\left(X \simeq X \otimes I \stackrel{\circledast \delta_{X}}{\longrightarrow} C_{1^{\prime} 1^{\prime \prime}} \otimes X_{1^{\prime \prime \prime}}=\bar{C} \otimes X\right)
\end{gathered}
$$

makes $\bar{C} \mathcal{V}$ into ${ }^{C} \mathcal{V} \otimes \mathcal{V}$.

2.6.2. Corollary. Let $\mathcal{A}$ be a $\mathbb{k}$-linear abelian essentially small category, let $\omega: \mathcal{A} \rightarrow$ $\mathcal{V}$ be a $\mathbb{k}$-linear exact faithful functor, and $\bar{C}=\int^{X \in \mathcal{A}} \omega X \otimes(\omega X)^{\vee}$. Then the category of $\bar{C}$-comodules ${ }^{\bar{C}} \mathcal{V}$ is equivalent to $\mathcal{A} \otimes \mathcal{V}$

This is closely related with results of Pareigis [6].

3. Squared bicoalgebras. Before we discuss bicoalgebras, let us consider the operation of tensor product of squared coalgebras. Notice that a braiding in $\mathcal{V}$ is not required.

3.1. Tensor product of squared coalgebras. Recall that $\mathcal{V} \otimes \mathcal{V}$ has a rigid monoidal structure $(A \bar{\otimes} B)_{12}=A_{1^{\prime} 2^{\prime \prime}} \otimes B_{1^{\prime \prime} 2^{\prime}}$ (Theorem 1.5.1).

3.1.1. Proposition. Let $A, B \in \operatorname{Coalgsq}(\widehat{\mathcal{V}})$. The tensor product $A \bar{\otimes} B$ has a coalgebra structure

$\Delta^{A \bar{\otimes} B}: A_{1^{\prime} 3^{\prime \prime}} \otimes B_{1^{\prime \prime} 3^{\prime}} \odot I_{2} \stackrel{A_{1^{\prime} 3^{\prime \prime}} \otimes \Delta_{1^{\prime \prime} 23^{\prime}}}{\longrightarrow} A_{1^{\prime} 3^{\prime \prime}} \otimes B_{1^{\prime \prime} 2^{\prime}} \otimes B_{2^{\prime \prime} 3^{\prime}} \simeq$

$A_{1^{\prime} 3^{\prime \prime}} \otimes B_{1^{\prime \prime} 2^{\prime}} \otimes I_{2^{\prime \prime}} \otimes B_{2^{\prime \prime \prime} 3^{\prime}} \stackrel{\Delta_{1^{\prime} 2^{\prime \prime} 3^{\prime \prime}} \otimes B_{1^{\prime \prime} 2^{\prime}} \otimes B_{2^{\prime \prime \prime} 3^{\prime}}}{\longrightarrow} A_{1^{\prime} 2^{\prime \prime}} \otimes B_{1^{\prime \prime} 2^{\prime}} \otimes A_{2^{\prime \prime \prime} 3^{\prime \prime}} \otimes B_{2^{4} 3^{\prime}}$,

$$
\varepsilon^{A \bar{\otimes} B}: A^{14} \otimes B^{23} \stackrel{A \otimes \varepsilon}{\longrightarrow} A^{13} \otimes I^{2} \simeq A^{12} \stackrel{\varepsilon}{\longrightarrow} I^{1} .
$$

The tensor product $A \bar{\otimes} B$ is associative and turn Coalgsq $(\widehat{\mathcal{V}})$ into a monoidal category.

Graphical notation and explanation of the comultiplication (3.1.1) and the counit (3.1.2) is the following

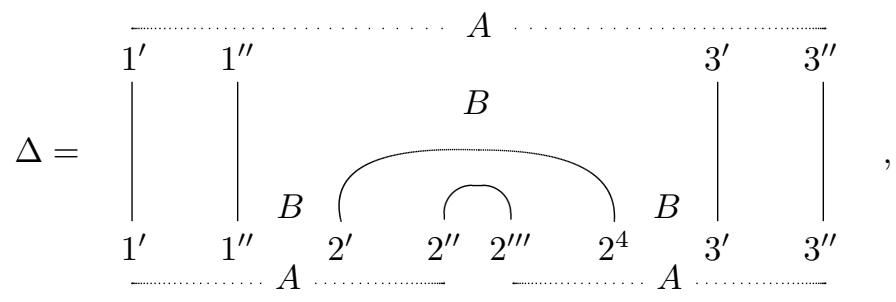




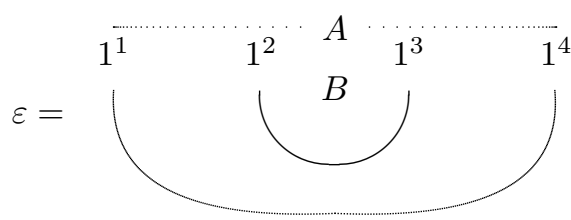

It reflects the canonical isomorphism $j_{+}: Y^{\vee} \otimes X^{\vee} \rightarrow(X \otimes Y)^{\vee}$ which is described in Section 1.2 on page 116 .

3.1.2. Remark. Let $A=X \odot X^{\vee}, B=Y \odot Y^{\vee}$. Then $A \bar{\otimes} B=(X \otimes Y) \odot\left(Y^{\vee} \otimes\right.$ $\left.X^{\vee}\right) \stackrel{1 \odot j_{+}}{\longrightarrow}(X \otimes Y) \odot(X \otimes Y)^{\vee}$ is a squared coalgebra isomorphism.

3.1.3. Proposition. Let $M \in{ }^{A} \widehat{\mathcal{V}}, N \in{ }^{B} \widehat{\mathcal{V}}$. Then $M \otimes N$ has the structure of an $A \bar{\otimes} B$-comodule

$$
\begin{aligned}
\delta^{M \otimes N} & =\left(M_{1^{\prime}} \otimes N_{1^{\prime \prime}} \odot I_{2} \stackrel{M \otimes \delta^{N}}{\longrightarrow} M_{1^{\prime}} \otimes B_{1^{\prime \prime} 2^{\prime}} \otimes N_{2^{\prime \prime}}\right. \\
& \left.\simeq M_{1^{\prime}} \otimes B_{1^{\prime \prime} 2^{\prime}} \otimes I_{2^{\prime \prime}} \otimes N_{2^{\prime \prime \prime}} \stackrel{\delta^{M} \otimes B \otimes N}{\longrightarrow} A_{1^{\prime} 2^{\prime \prime}} \otimes B_{1^{\prime \prime} 2^{\prime}} \otimes M_{2^{\prime \prime \prime}} \otimes N_{2^{4}}\right) .
\end{aligned}
$$

For $L \in C \widehat{\mathcal{V}}$, both ways to construct an $A \bar{\otimes} B \bar{\otimes} C$-comodule structure on $M \otimes N \otimes L$ coincide.

Graphical notation for the coaction $\delta^{M \otimes N}$ is

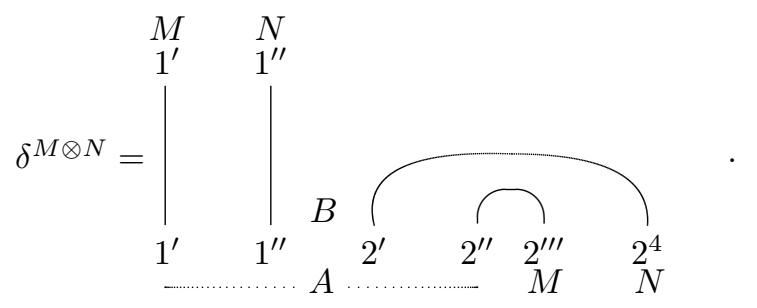

3.1.4. Remark. If $A=M \odot M^{\vee}, B=N \odot N^{\vee}$, the coaction $\delta^{M \otimes N}$ of $A \bar{\otimes} B$ on $M \otimes N$ is mapped by $1 \odot j_{+}$to the canonical coaction of $(M \otimes N) \odot(M \otimes N)^{\vee}$ (see Remark 3.1.2).

\subsection{Bicoalgebras}

3.2.1. Definition. A squared bicoalgebra $B=(B, \Delta, \varepsilon, m, \eta)$ in $\widehat{\mathcal{V}}$ is a squared coalgebra $(B, \Delta, \varepsilon) \in \operatorname{Coalgsq}(\widehat{\mathcal{V}})$ equipped with an algebra structure $(B, m, \eta)$ in $(\widehat{\mathcal{V} \otimes \mathcal{V}}, \bar{\otimes})$ (such that the multiplication $m: B \bar{\otimes} B \rightarrow B \in \widehat{\mathcal{V} \otimes \mathcal{V}}$ is associative and $\eta: I \odot I \rightarrow B \in$ $\widehat{\mathcal{Q} \otimes \mathcal{V}}$ is the unit) and such that $m, \eta$ are squared coalgebra homomorphisms, that is,

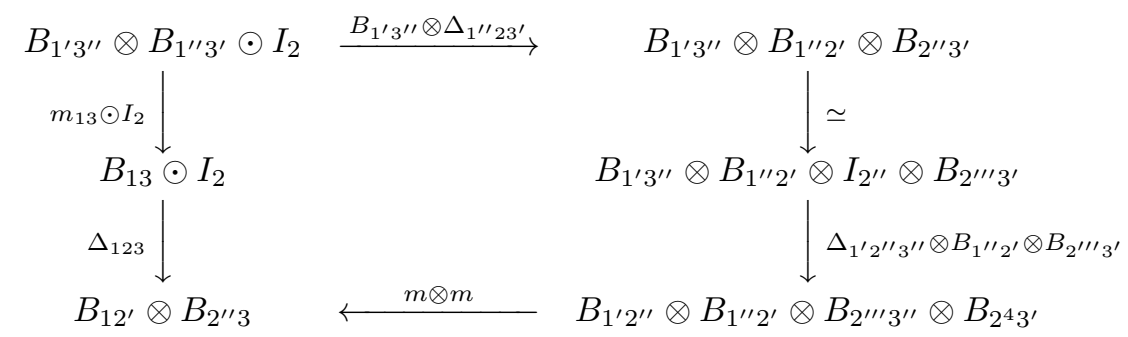




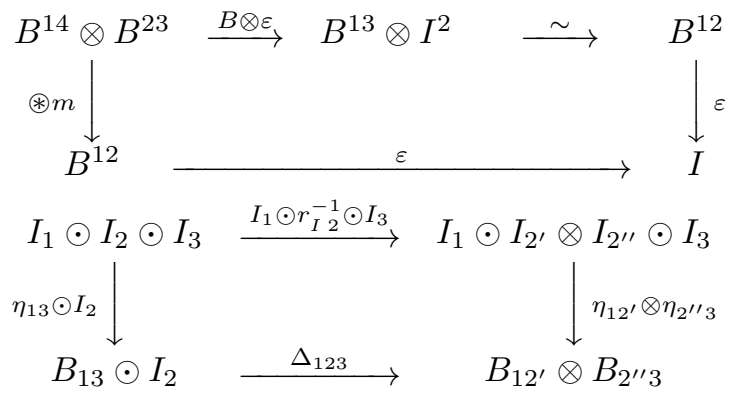

hold.

$(I \otimes I \stackrel{\circledast \eta}{\longrightarrow} \circledast B \stackrel{\varepsilon}{\rightarrow} I)=r_{I}$

Morphisms of bicoalgebras are those preserving algebra and squared coalgebra structures. The category of bicoalgebras in $\widehat{V}$ is denoted Bicoalg $(\widehat{\mathcal{V}})$.

3.2.2. Remark. Definition of a squared bicoalgebra is not self-dual. One can define dually squared bialgebras which are ordinary coalgebras and squared algebras.

For $M, N \in{ }^{B} \widehat{\mathcal{V}}$ let us define a $B$-comodule structure on $M \otimes N \in \widehat{\mathcal{V}}$ via (3.1.3):

$$
M_{1^{\prime}} \otimes N_{1^{\prime \prime}} \odot I_{2} \stackrel{\delta^{M \otimes N}}{\longrightarrow} B_{1^{\prime} 2^{\prime \prime}} \otimes B_{1^{\prime \prime} 2^{\prime}} \otimes M_{2^{\prime \prime}} \otimes N_{2^{4}} \stackrel{m \otimes M \otimes N}{\longrightarrow} B_{12^{\prime}} \otimes M_{2^{\prime \prime}} \otimes N_{2^{\prime \prime \prime}} .
$$

Proposition 3.1.3 shows that the tensor product of $B$-comodules is associative. The associativity isomorphism coincides with that one of $(\widehat{\mathcal{V}}, \otimes)$. Therefore, the category $(B \widehat{\mathcal{V}}, \otimes)$ is monoidal with the unit object $\left(I, \delta_{I}\right)$,

$$
\delta_{I}=\left(I_{1} \odot I_{2} \stackrel{\eta}{\rightarrow} B_{12} \simeq B_{12^{\prime}} \otimes I_{2^{\prime \prime}}\right) .
$$

\subsection{Monoidal reconstruction}

3.3.1. Definition. Let $(\mathcal{A}, \otimes)$ be a $\mathbb{k}$-linear abelian monoidal essentially small category. A monoidal fibre functor is a $\mathbb{k}$-linear exact faithful monoidal functor $\left(\omega_{\mathcal{A}}, \omega^{\mathcal{A}}\right)$ : $\mathcal{A} \rightarrow \mathcal{V}$. Let the category of monoidal fibre functors $\mathfrak{M}(\mathcal{V})$ have monoidal fibre functors as objects and let morphisms from $\left(\omega_{\mathcal{A}}, \omega^{\mathcal{A}}\right): \mathcal{A} \rightarrow \mathcal{V}$ to $\left(\omega_{\mathcal{B}}, \omega^{\mathcal{B}}\right): \mathcal{B} \rightarrow \mathcal{V}$ be equivalence classes of triples $(F, f, \phi)$, where $(F, f): \mathcal{A} \rightarrow \mathcal{B}$ is a monoidal functor and $\phi:\left(\omega_{\mathcal{A}}, \omega^{\mathcal{A}}\right) \rightarrow\left(\omega_{\mathcal{B}}, \omega^{\mathcal{B}}\right) \circ(F, f)$ is an isomorphism of monoidal functors. Two such triples $(F, f, \phi)$ and $(G, g, \gamma)$ are equivalent if there is a functorial isomorphism $\zeta: F \rightarrow G$ such that (2.4.1) holds. The composite of two morphisms represented by $(F, f, \phi)$ and $(G, g, \gamma)$ is represented by $\left(G \circ F, G f \circ g_{F, F}, \gamma_{F} \circ \phi\right)$.

The functor $F$ from the above triple is exact and faithful. Forgetting the monoidal structure we get a functor $\mathfrak{M}(\mathcal{V}) \rightarrow \mathfrak{A}(\mathcal{V})$. It is faithful since $\omega_{\mathcal{B}} f_{X, Y}$ is determined uniquely by given $F, \phi$ from the condition $\phi:\left(\omega_{\mathcal{A}}, \omega^{\mathcal{A}}\right) \stackrel{\sim}{\rightarrow}\left(\omega_{\mathcal{B}}, \omega^{\mathcal{B}}\right) \circ(F, f)$, and $\omega_{\mathcal{B}}$ is faithful.

There is a functor $\Phi: \operatorname{Bicoalg}(\widehat{\mathcal{V}}) \rightarrow \mathfrak{M}(\mathcal{V}), B \mapsto\left((\mathcal{U}, \mathrm{id}):{ }^{B} \mathcal{V} \rightarrow \mathcal{V}\right)$. We want to prove that this is an equivalence.

Let $\left(\omega_{\mathcal{A}}, \omega^{\mathcal{A}}\right):(\mathcal{A}, \otimes) \rightarrow(\mathcal{V}, \otimes)$ be a monoidal fibre functor. Assume that $C_{\mathcal{A}}$ is the coend (2.4.2) constructed from the functor $\omega_{\mathcal{A}}$ (classifying coalgebra of the category $\mathcal{A}$ ). Then, $\mathcal{A} \simeq{ }^{C_{\mathcal{A}}} \mathcal{V}$. 
3.3.2. TheOREM. (a) The squared coalgebra $C_{\mathcal{A}}$ is a bicoalgebra. The monoidal functor $\omega_{\mathcal{A}}$ admits the factorisation

$$
\left(\omega_{\mathcal{A}}, \omega^{\mathcal{A}}\right)=\left(\mathcal{A} \stackrel{\left(F_{\mathcal{A}}, f^{\mathcal{A}}\right)}{\longrightarrow} C_{\mathcal{A}} \mathcal{V} \stackrel{(\mathcal{U}, \mathrm{id})}{\longrightarrow} \mathcal{V}\right)
$$

where $F_{\mathcal{A}}$ is the equivalence from Theorem 2.5.1 and $f_{X, Y}^{\mathcal{A}}=\omega_{X, Y}^{\mathcal{A}}$.

(b) The correspondence $\left(\omega_{\mathcal{A}}, \omega^{\mathcal{A}}\right) \mapsto C_{\mathcal{A}}$ extends to a functor

$$
\Psi: \mathfrak{M}(\mathcal{V}) \rightarrow \operatorname{Bicoalg}(\widehat{\mathcal{V}}) .
$$

3.3.3. Corollary. The monoidal functor $\left(F_{\mathcal{A}}, f^{\mathcal{A}}\right): \mathcal{A} \rightarrow \Phi(\Psi(\mathcal{A}))={ }^{C_{\mathcal{A}}} \mathcal{V}$ constructed in Theorem 3.3.2 together with id $:\left(\omega_{\mathcal{A}}, \omega^{\mathcal{A}}\right) \rightarrow(\mathcal{U}, \mathrm{id}) \circ\left(F_{\mathcal{A}}, f^{\mathcal{A}}\right)$ gives an isomorphism of functors

$$
F: \operatorname{Id}_{\mathfrak{M}(\mathcal{V})} \stackrel{\sim}{\longrightarrow} \Phi \Psi .
$$

P r o of. We already know by Theorem 2.5.2 that $F$ is an isomorphism of the forgetful functor $\mathfrak{M}(\mathcal{V}) \rightarrow \mathfrak{A}(\mathcal{V})$ with $\Phi \Psi: \mathfrak{M}(\mathcal{V}) \rightarrow \mathfrak{M}(\mathcal{V}) \rightarrow \mathfrak{A}(\mathcal{V})$. Theorem 3.3.2 implies that this isomorphism is in $\mathfrak{M}(\mathcal{V})$.

3.3.4. TheOrEm. The functors $\Phi: \operatorname{Bicoalg}(\widehat{\mathcal{V}}) \rightarrow \mathfrak{M}(\mathcal{V}), B \mapsto^{B} \mathcal{V}$ and $\Psi: \mathfrak{M}(\mathcal{V}) \rightarrow$ $\operatorname{Bicoalg}(\widehat{\mathcal{V}}),\left(\omega_{\mathcal{A}}, \omega^{\mathcal{A}}\right) \mapsto C_{\mathcal{A}}$ are equivalences, quasiinverse to each other.

P r o o f. By Theorem 3.3.2 $\Phi$ is essentially surjective on objects. Corollary 3.3.3 implies that $\Phi$ is full. By Theorem 2.5.2 $\Phi$ is faithful, hence, $\Phi$ is an equivalence. $\Psi$ is quasi-inverse to $\Phi$ by Corollary 3.3.3.

In the case $\mathcal{V}=\mathbb{k}$-vect this theorem was proved by Schauenburg [8].

To construct explicitly the isomorphism $\Psi \Phi \rightarrow \operatorname{Id}_{\mathfrak{M}(\mathcal{V})}$ let us use the results for $\mathfrak{A}(\mathcal{V})$. Let $B$ be a bicoalgebra in $\widehat{\mathcal{V}}$, let $\mathcal{A}={ }^{B} \mathcal{V}$, let $\omega_{\mathcal{A}}=\mathcal{U}:{ }^{B} \mathcal{V} \rightarrow \mathcal{V}$ and $\omega^{\mathcal{A}}=$ id. It was shown in Proposition 2.5.3 that there is a unique coalgebra isomorphism $h_{B}: C_{\mathcal{A}}=\Psi \Phi(B) \rightarrow B$ such that for any $X \in{ }^{B} \mathcal{V}$ the composite $X \odot X^{\vee} \stackrel{i_{X}}{\longrightarrow} C_{\mathcal{A}} \stackrel{h_{B}}{\longrightarrow} B$ is the canonical coalgebra morphism (2.5.1).

3.3.5. Proposition (bicoalgebra reconstruction). The morphism $h_{B}: C_{\mathcal{A}} \rightarrow B$ is an isomorphism of bicoalgebras giving the functorial isomorphism

$$
h: \Psi \Phi \rightarrow \operatorname{Id}_{\operatorname{Bicoalg}(\widehat{\mathcal{V}})} .
$$

3.4. Relationship with braided bialgebras. Let us consider the case of braided $\mathcal{V}$. Then it makes sense to consider braided (quasiclassical) bialgebras.

There is a unique functorial isomorphism $\phi:(\circledast X) \otimes(\circledast Y) \rightarrow \circledast(X \bar{\otimes} Y), X, Y \in \mathcal{V} \otimes \mathcal{V}$, such that for $X=A \odot B, Y=C \odot D$ it equals

$$
\begin{gathered}
\phi=(432)_{+} \sim(A \otimes B) \otimes(C \otimes D) \rightarrow(A \otimes C) \otimes(D \otimes B), \\
\phi=
\end{gathered}
$$

Indeed, it extends uniquely to arbitrary $X, Y \in \mathcal{V} \otimes \mathcal{V}$ via resolutions

$$
0 \rightarrow M \stackrel{i}{\longrightarrow} A \odot B \stackrel{\sum f_{l} \odot g_{l}}{\longrightarrow} C \odot D .
$$


3.4.1. Proposition. When $\mathcal{V}$ is braided, there is a monoidal functor

$$
\left(\circledast, \phi, r_{I}^{-1}\right):(\mathcal{V} \otimes \mathcal{V}, \bar{\otimes}, I \odot I) \rightarrow(\mathcal{V}, \otimes, I) .
$$

3.4.2. Proposition. Let $B=(B, \Delta, \varepsilon, m, \eta)$ be a bicoalgebra in $\widehat{\mathcal{V}}$. Denote

$$
\begin{aligned}
\bar{B} & =\circledast B=B^{12}, \\
\bar{\Delta} & =\left(B^{12} \simeq B^{13} \otimes I^{2} \stackrel{\Delta^{123}}{\longrightarrow} B^{12} \otimes B^{34}\right), \\
\bar{\eta} & =\left(I \stackrel{r_{I}^{-1}}{\longrightarrow} I \otimes I \stackrel{\circledast \eta}{\longrightarrow} \circledast B\right), \\
\bar{m} & =\left(B^{12} \otimes B^{34} \stackrel{\phi}{\longrightarrow} B^{14} \otimes B^{23} \stackrel{\circledast m}{\longrightarrow} B^{12}\right) .
\end{aligned}
$$

Then $(\bar{B}, \bar{\Delta}, \varepsilon, \bar{m}, \bar{\eta})$ is a braided bialgebra in $\widehat{\mathcal{V}}$.

3.4.3. Proposition. There exists a $\mathbb{k}$-linear exact faithful monoidal functor

$$
\begin{gathered}
(F, \text { id, id }):{ }^{B} \mathcal{V} \rightarrow{ }^{\bar{B}} \mathcal{V}, \quad F\left(X, \delta_{X}\right)=\left(X, \bar{\delta}_{X}\right), \quad F(f)=f, \\
\bar{\delta}_{X}=\left(X \stackrel{r_{X}^{-1}}{\longrightarrow} X \otimes I \stackrel{\circledast \delta_{X}}{\longrightarrow} \bar{B} \otimes X\right),
\end{gathered}
$$

commuting with the underlying functor.

4. Hopf coalgebras. In addition to standard assumptions of Section 1.3 we assume that $X^{\vee \vee}$ is functorially isomorphic to $X \in \mathcal{V}$. Let us call such categories rigid-involutive. In particular, braided categories are rigid-involutive. However, we don't need the braiding in $\mathcal{V}$ and our study of Hopf algebras applies to non-braided $\mathcal{V}$ as well.

Let us pick a functorial isomorphism $\zeta_{X}: X \rightarrow X^{\vee \vee}$. Our notions will explicitly depend on the choice of $\zeta$. Different choices lead to isomorphic constructions.

\subsection{Opposite coalgebras}

4.1.1. Proposition. Let $p: \mathcal{P} \rightarrow \mathcal{V}$ be a functor and let $p^{\vee}: \mathcal{P}^{\text {op }} \rightarrow \mathcal{V}$ be the composite functor, $p^{\vee}=\vee \circ p$, that is, $p^{\vee}(X)=p(X)^{\vee}, p^{\vee}(f: X \rightarrow Y)=\left(p(f)^{t}: p(Y)^{\vee} \rightarrow p(X)^{\vee}\right)$. Let $C_{p}$ and $C_{p} \vee$ be the corresponding squared coalgebras. Then there is an isomorphism $z: P C_{p} \rightarrow C_{p^{\vee}} \in \widehat{\mathcal{V} \otimes \mathcal{V}}$, which satisfies the following diagram

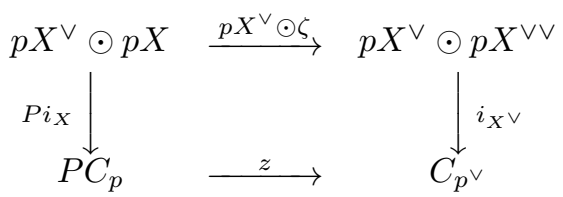

for each $X \in \mathrm{Ob} \mathcal{P}$. There are unique coalgebra structures $\left(p X^{\vee} \odot p X, \Delta^{\mathrm{op}}, \varepsilon^{\mathrm{op}}\right)$ and $\left(P C_{p}, \Delta^{\mathrm{op}}, \varepsilon^{\mathrm{op}}\right)$ such that each morphism in diagram (4.1.1) is a coalgebra morphism. For $M=p X$ this is

$$
\begin{gathered}
\Delta^{\mathrm{op}}: M^{\vee} \odot I \odot M \stackrel{1 \odot \operatorname{coev} \odot 1}{\longrightarrow} M^{\vee} \odot M^{\vee \vee} \otimes M^{\vee} \odot M \\
\stackrel{1 \odot \zeta^{-1} \otimes 1 \odot 1}{\longrightarrow} M^{\vee} \odot M \otimes M^{\vee} \odot M, \\
\varepsilon^{\mathrm{op}}: M^{\vee} \otimes M \stackrel{1 \otimes \zeta}{\longrightarrow} M^{\vee} \otimes M^{\vee \vee} \stackrel{\mathrm{ev}}{\longrightarrow} I .
\end{gathered}
$$

4.1.2. Definition. Let $C$ be a squared coalgebra in $\widehat{\mathcal{V}}$. The opposite coalgebra $C_{\text {op }}=$ $\left(P C, \Delta^{\mathrm{op}}, \varepsilon^{\mathrm{op}}\right)$ is the unique coalgebra structure on $P C$ such that $P \ddot{\imath}_{M}: M^{\vee} \odot M \rightarrow P C$ 
is a squared coalgebra homomorphism for any $C$-comodule $M \in{ }^{C} \mathcal{V}$, where $M^{\vee} \odot M$ is equipped with coalgebra structure (4.1.2), (4.1.3).

To check the existence of the opposite coalgebra notice that any coalgebra has the form $C_{p}$ for some $\mathcal{P} \subset{ }^{C} \mathcal{V}$ and apply Proposition 4.1.1.

4.1.3. Remark. The duality yields an equivalence of categories $\left({ }^{C} \mathcal{V}\right)^{\mathrm{op}} \rightarrow{ }^{C_{\mathrm{op}}} \mathcal{V}$, $\left(M, \delta_{M}\right) \mapsto\left(M^{\vee}, \delta_{M^{\vee}}^{\prime}\right)$, where $\delta^{\prime}$ is given by

$$
\begin{aligned}
\delta_{M^{\vee}}^{\prime}=\left(M^{\vee} \odot I \stackrel{M^{\vee} \odot \text { coev }}{\longrightarrow}\right. & M^{\vee} \odot M^{\vee \vee} \otimes M^{\vee} \stackrel{M^{\vee} \odot \zeta^{-1} \otimes M^{\vee}}{\longrightarrow} \\
& \left.M^{\vee} \odot M \otimes M^{\vee} \stackrel{P i_{M} \otimes M^{\vee}}{\longrightarrow}(P C)_{12^{\prime}} \otimes M_{2^{\prime \prime}}^{\vee}\right) .
\end{aligned}
$$

This follows by Proposition 4.1.1.

Clearly, another choice of $\zeta$ gives an isomorphic coalgebra structure in $P C$.

4.1.4. Proposition. Each functorial isomorphism $\zeta: X \rightarrow X^{\vee \vee}$ determines a functor

$$
\begin{gathered}
P_{\zeta}: \operatorname{Coalgsq}(\widehat{\mathcal{V}}) \rightarrow \operatorname{Coalgsq}(\widehat{\mathcal{V}}), \quad h \mapsto P h, \\
P_{\zeta}(C)=C_{\mathrm{op}}=\left(P C, \Delta^{\mathrm{op}}, \varepsilon^{\mathrm{op}}\right)=\left(P C, \Delta^{\zeta}, \varepsilon^{\zeta}\right) .
\end{gathered}
$$

All such functors are isomorphic.

4.2. Comparison with opposite coalgebra in braided case. If $\mathcal{V}$ is braided, we have the usual notion of an opposite coalgebra. The following proposition shows that the new notion of opposite coincides with traditional one at the quasiclassical level.

4.2.1. Proposition. Let $C$ be a squared coalgebra in $\widehat{\mathcal{V}}$, let $\zeta=u_{1}^{2}: X \rightarrow X^{\vee \vee}$ and let $\bar{C}_{\mathrm{op}}=\left(\bar{C}, \bar{\Delta}^{\mathrm{op}}\right)$ be the quasiclassical opposite to $\bar{C}$ :

$$
\bar{\Delta}^{\mathrm{op}}=(\bar{C} \stackrel{\bar{\Delta}}{\rightarrow} \bar{C} \otimes \bar{C} \stackrel{c}{\rightarrow} \bar{C} \otimes \bar{C}) .
$$

Then the isomorphism, induced by the braiding

$$
c: \bar{C}_{\mathrm{op}}=\left(C^{12}, \bar{\Delta}^{\mathrm{op}}, \varepsilon\right) \rightarrow\left(C^{21}, \overline{\Delta^{\mathrm{op}}}, \varepsilon^{\mathrm{op}}\right)=\overline{C_{\mathrm{op}}}
$$

is a coalgebra isomorphism.

4.3. The antipode. Hopf coalgebras are bicoalgebras, whose categories of comodules are rigid. However, at the moment we use another definition: Hopf coalgebras are bicoalgebras with an antipode. The first definition will be made a result.

4.3.1. Definition. Let $H$ be a bicoalgebra in $\widehat{\mathcal{V}}$. A right antipode in $H$ (with respect to $\zeta$ ) is a morphism $\gamma^{\prime}=\gamma_{\zeta}: H_{\mathrm{op}} \rightarrow H \in \widehat{\mathcal{V} \otimes \mathcal{V}}$ such that

$$
\begin{aligned}
& \left(H_{\mathrm{op} 1^{\prime \prime} 1^{\prime}} \odot I_{2} \stackrel{\Delta_{1^{\prime \prime} 21^{\prime}}^{\mathrm{op}}}{\longrightarrow} H_{\mathrm{op} 1^{\prime \prime} 2^{\prime}} \otimes H_{\mathrm{op} 2^{\prime \prime} 1^{\prime}}=H_{1^{\prime} 2^{\prime \prime}} \otimes H_{\mathrm{op} 1^{\prime \prime} 2^{\prime}}\right. \\
& \left.\stackrel{H \otimes \gamma_{1^{\prime \prime} 2^{\prime}}^{\prime}}{\longrightarrow} H_{1^{\prime} 2^{\prime \prime}} \otimes H_{1^{\prime \prime} 2^{\prime}} \stackrel{m}{\longrightarrow} H_{12}\right) \\
& =\left(H_{1^{\prime} 1^{\prime \prime}} \odot I_{2} \stackrel{\varepsilon \odot I}{\longrightarrow} I_{1} \odot I_{2} \stackrel{\eta}{\longrightarrow} H_{12}\right),
\end{aligned}
$$




$$
\begin{aligned}
& \left(I_{1} \odot H_{2^{\prime \prime} 2^{\prime}} \stackrel{\Delta_{2^{\prime \prime} 12^{\prime}}}{\longrightarrow} H_{2^{\prime \prime} 1^{\prime}} \otimes H_{1^{\prime \prime} 2^{\prime}}=H_{\mathrm{op} 1^{\prime} 2^{\prime \prime}} \otimes H_{1^{\prime \prime} 2^{\prime}}\right. \\
& \left.\stackrel{\gamma_{1^{\prime} 2^{\prime \prime}}^{\prime} \otimes H}{\longrightarrow} H_{1^{\prime} 2^{\prime \prime}} \otimes H_{1^{\prime \prime} 2^{\prime}} \stackrel{m}{\longrightarrow} H_{12}\right) \\
& =\left(I_{1} \odot H_{\mathrm{op} 2^{\prime} 2^{\prime \prime}} \stackrel{I \odot \varepsilon^{\mathrm{op}}}{\longrightarrow} I_{1} \odot I_{2} \stackrel{\eta}{\rightarrow} H_{12}\right) .
\end{aligned}
$$

A left antipode in $H$ (with respect to $\zeta$ ) is a morphism ${ }^{\prime} \gamma={ }_{\zeta} \gamma: H_{\mathrm{op}} \rightarrow H \in \widehat{\mathcal{V} \otimes} \mathcal{V}$ such that

$$
\begin{aligned}
& \left(H_{1^{\prime \prime} 1^{\prime}} \odot I_{2} \stackrel{\Delta_{1^{\prime \prime} 21^{\prime}}}{\longrightarrow} H_{1^{\prime \prime} 2^{\prime}} \otimes H_{2^{\prime \prime} 1^{\prime}}=H_{\mathrm{op} \mathrm{1} 2^{\prime \prime}} \otimes H_{1^{\prime \prime 2} 2^{\prime}}\right. \\
& \left.\stackrel{\prime}{\stackrel{\gamma_{1^{\prime} 2^{\prime \prime}} \otimes H}{\longrightarrow}} H_{1^{\prime} 2^{\prime \prime}} \otimes H_{1^{\prime \prime} 2^{\prime}} \stackrel{m}{\longrightarrow} H_{12}\right) \\
& =\left(H_{\mathrm{op} 1^{\prime} 1^{\prime \prime}} \odot I_{2} \stackrel{\varepsilon^{\mathrm{op}} \odot I_{2}}{\longrightarrow} I_{1} \odot I_{2} \stackrel{\eta}{\rightarrow} H_{12}\right), \\
& \left(I_{1} \odot H_{\mathrm{op} 2^{\prime \prime} 2^{\prime}} \stackrel{\Delta_{2^{\prime \prime} 1^{\prime}}^{\mathrm{op}}}{\longrightarrow} H_{\mathrm{op} 2^{\prime \prime} 1^{\prime}} \otimes H_{\mathrm{op} \mathrm{1} 2^{\prime \prime} 2^{\prime}}=H_{1^{\prime} 2^{\prime \prime}} \otimes H_{\mathrm{op} 1^{\prime \prime} 2^{\prime}}\right. \\
& \left.\stackrel{H \otimes^{\prime} \gamma_{1^{\prime \prime} 2^{\prime}}}{\longrightarrow} H_{1^{\prime} 2^{\prime \prime}} \otimes H_{1^{\prime \prime} 2^{\prime}} \stackrel{m}{\longrightarrow} H_{12}\right) \\
& =\left(I_{1} \odot H_{2^{\prime} 2^{\prime \prime}} \stackrel{I \odot \varepsilon}{\longrightarrow} I_{1} \odot I_{2} \stackrel{\eta}{\longrightarrow} H_{12}\right) .
\end{aligned}
$$

A (squared) Hopf coalgebra is a bicoalgebra which has a right and a left antipode.

Graphical expression of these equations is the following. Here $X$ is an $H$-comodule and $\ddot{\imath}_{X}: X \odot X^{\vee} \rightarrow H$ is implicit.
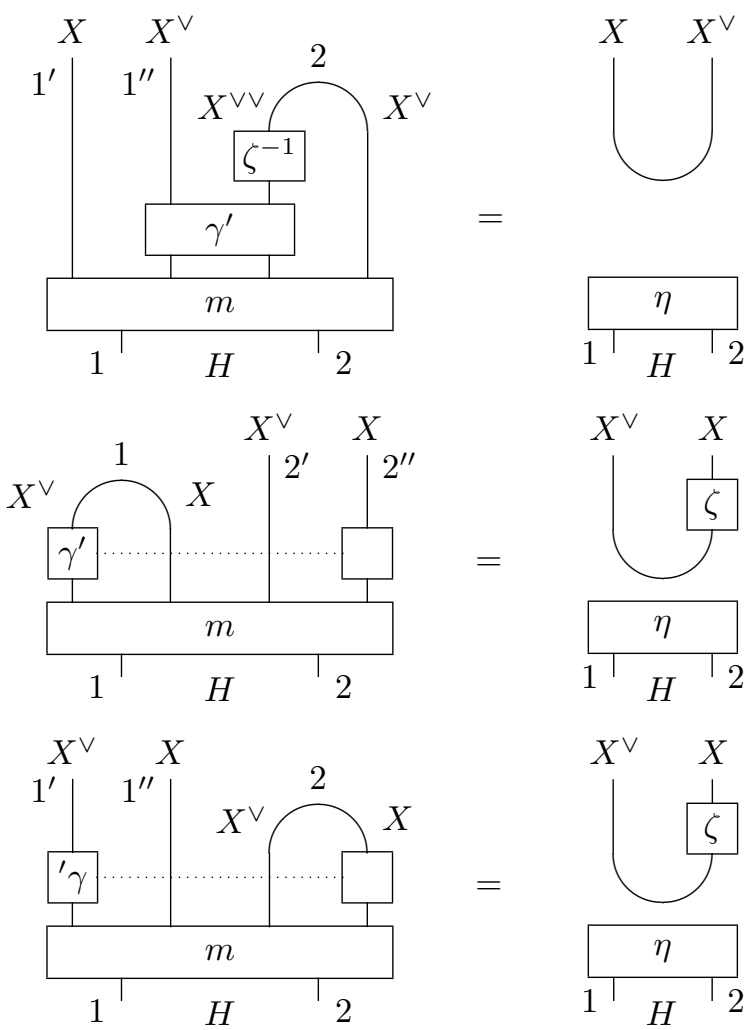

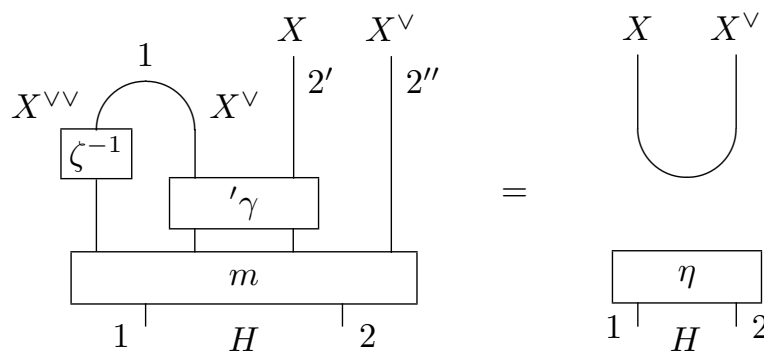

$=$

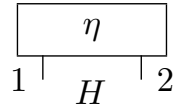

The dual notion is called squared Hopf algebra.

4.3.2. Proposition. Let $\left(H, \gamma^{\prime},{ }^{\prime} \gamma\right)$ be a Hopf coalgebra. Then $\gamma^{\prime},^{\prime} \gamma: H_{\mathrm{op}} \rightarrow H$ are homomorphisms of squared coalgebras.

4.3.3. ThEOREM. The category ${ }^{H} \mathcal{V}$ of comodules over a Hopf coalgebra $H$ is rigid. For the right dual of $X$ one can take

$$
\left(X^{\vee}, \delta_{X^{\vee}}: X_{1}^{\vee} \odot I_{2} \stackrel{\delta_{X}^{\prime} \vee}{\longrightarrow} H_{\mathrm{op} 12^{\prime}} \otimes X_{2^{\prime \prime}}^{\vee} \stackrel{\gamma_{12^{\prime}}^{\prime} \otimes X_{2^{\prime \prime}}^{\vee}}{\longrightarrow} H_{12^{\prime}} \otimes X_{2^{\prime \prime}}^{\vee}\right),
$$

for the left dual -

$$
\left({ }^{\vee} X, \delta \vee_{X}:{ }^{\vee} X_{1} \odot I_{2} \stackrel{{ }^{\prime} \delta \vee_{X}}{\longrightarrow} H_{\mathrm{op} 12^{\prime}} \otimes{ }^{\vee} X_{2^{\prime \prime}} \stackrel{{ }^{\prime} \gamma_{12^{\prime}} \otimes{ }^{\vee} X_{2^{\prime \prime}}}{\longrightarrow} H_{12^{\prime}} \otimes{ }^{\vee} X_{2^{\prime \prime}}\right) .
$$

The evaluation and coevaluation morphisms are the same as in $\mathcal{V}$.

The formulae

$$
\begin{aligned}
& \delta_{X^{\vee}}=\left(X^{\vee} \odot I \stackrel{X^{\vee} \odot \text { coev }}{\longrightarrow} X^{\vee} \odot X^{\vee \vee} \otimes X^{\vee} \stackrel{X^{\vee} \otimes \zeta^{-1} \otimes X^{\vee}}{\longrightarrow} X_{1}^{\vee} \odot X_{2^{\prime}} \otimes X_{2^{\prime \prime}}^{\vee}\right. \\
& \left.\stackrel{\ddot{i}_{2^{\prime} 1} \otimes X^{\vee}}{\longrightarrow} H_{\mathrm{op} 12^{\prime}} \otimes X_{2^{\prime \prime}}^{\vee} \stackrel{\gamma^{\prime} \otimes X^{\vee}}{\longrightarrow} H_{12^{\prime}} \otimes X_{2^{\prime \prime}}^{\vee}\right), \\
& \delta^{\vee_{X}}=\left({ }^{\vee} X \odot I \stackrel{{ }^{\vee} X \odot \operatorname{coev}^{\vee}}{\longrightarrow} X \odot X \otimes{ }^{\vee} X \stackrel{{ }^{t} \zeta^{-1} \odot X \otimes{ }^{\vee} X}{\longrightarrow} X_{1}^{\vee} \odot X_{2^{\prime}} \otimes{ }^{\vee} X_{2^{\prime \prime}}\right. \\
& \left.\stackrel{\ddot{i}_{2^{\prime} 1} \otimes^{\vee} X}{\longrightarrow} H_{\mathrm{op} 12^{\prime}} \otimes{ }^{\vee} X_{2^{\prime \prime}} \stackrel{{ }^{\prime} \gamma \otimes^{\vee} X}{\longrightarrow} H_{12^{\prime}} \otimes{ }^{\vee} X_{2^{\prime \prime}}\right) \text {. }
\end{aligned}
$$

together with definition (2.5.1) of $\ddot{i}_{X}$ imply

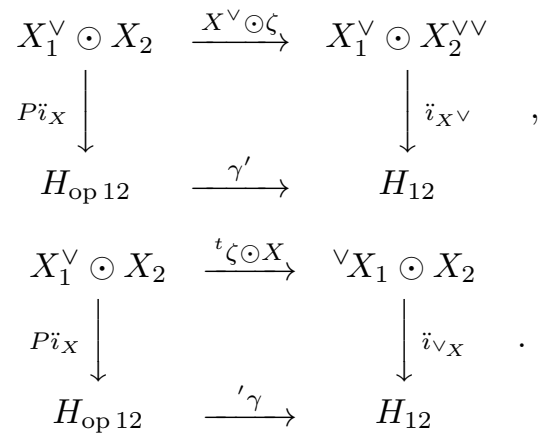

4.3.4. Corollary. The right and the left antipodes $\gamma^{\prime},{ }^{\prime} \gamma: H_{\mathrm{op}} \rightarrow H$ of a Hopf coalgebra are unique and invertible.

Uniqueness follows from the fundamental theorem on coalgebras (Corollary 2.5.4) and diagrams (4.3.3), (4.3.4). Invertibility follows from the fact that $X \mapsto X^{\vee}, X \mapsto{ }^{\vee} X$ are equivalences. If only right antipode exists for $H$, it need not be invertible. 
4.3.5. Remark. The property of being a Hopf coalgebra does not depend on the choice of $\zeta$.

4.3.6. Proposition. Let $H$ be a bicoalgebra in $\widehat{v}$.

(a) If $H$ has a right antipode $\gamma_{\zeta}: H_{\mathrm{op}} \rightarrow H$ with respect to $\zeta$ and it is invertible, then ${ }^{t} \zeta^{-1} \gamma=P \gamma_{\zeta}^{-1}$ is a left antipode for $H$ with respect to ${ }^{t} \zeta^{-1}$.

(b) If $H$ has a left antipode $\zeta \gamma: H_{\mathrm{op}} \rightarrow H$ with respect to $\zeta$ and it is invertible, then $\gamma^{t} \zeta^{-1}=P_{\zeta} \gamma^{-1}$ is a right antipode for $H$ with respect to ${ }^{t} \zeta^{-1}$

In both cases $H$ is a Hopf coalgebra.

4.4. Comparison with braided Hopf algebras. We recall that if $\mathcal{V}$ is braided, the tensor functor $\left(\circledast, \phi, r_{I}^{-1}\right): \mathcal{V} \mathcal{V} \rightarrow \mathcal{V}$ from Proposition 3.4.1 transforms squared bicoalgebras $B$ into quasiclassical bialgebras $\bar{B}$ (see Proposition 3.4.2).

4.4.1. Proposition. Let $H$ be a squared Hopf algebra in $\widehat{\mathcal{V}}$. Then $\bar{H}$ is a quasiclassical Hopf algebra in $\widehat{\mathcal{V}}$. If $\gamma^{\prime}$ is the right antipode for $H$ with respect to $\zeta=u_{1}^{2}$, then

$$
\gamma_{\bar{H}}=\left(H^{12} \stackrel{c}{\rightarrow} H^{21} \stackrel{\circledast \gamma^{\prime}}{\longrightarrow} H^{12}\right)
$$

is the antipode for $\bar{H}$. If $\gamma$ is the left antipode for $H$ with respect to $\tilde{\zeta}=u_{-1}^{2}$, then

$$
\tilde{\gamma}_{\bar{H}}=\gamma_{\bar{H}}^{-1}=\left(H^{12} \stackrel{c^{-1}}{\longrightarrow} H^{21} \stackrel{\circledast}{\stackrel{\prime}{\longrightarrow}} H^{12}\right)
$$

is the skew antipode for $\bar{H}$.

5. Quasitriangular Hopf coalgebras. We want to discuss braided monoidal categories. Naturally, we assume that $\mathcal{V}$ is braided. It was shown in Propositions 3.4.1, 3.4.2 that a monoidal functor $\left(\circledast, \phi, r_{I}^{-1}\right):(\mathcal{V} \otimes \mathcal{V}, \bar{\otimes}, I \odot I) \rightarrow(\mathcal{V}, \otimes, I)$ maps bicoalgebras $H$ to braided bialgebras $\bar{H}=(\circledast H, \bar{\Delta}, \varepsilon, \bar{m}, \bar{\eta})$. The structure responsible for the braiding in ${ }^{B} \mathcal{V}$ is the $R$-matrix. Bicoalgebras which admit an $R$-matrix will be called quasitriangular. We are going to establish correspondence between $\mathbb{k}$-linear exact monoidal functors $\omega: \mathcal{C} \rightarrow \mathcal{V}$ which do not preserve braiding and quasitriangular bicoalgebras. Quasitriangular Hopf coalgebra is a natural implementation of the idea of a quantum braided group [4]. Although there are two $R$-matrices $-R_{+}$and $R_{-}$, they are linearly expressed one through another with the help of braiding in $\mathcal{V}$.

\subsection{R-matrices in Hopf coalgebras}

5.1.1. Definition. A quasitriangular Hopf coalgebra in $\widehat{\mathcal{V}}$ is a Hopf coalgebra $H$ in $\widehat{\mathcal{V}}$ equipped with bilinear forms $R_{+}: \bar{H} \otimes \bar{H} \rightarrow I, R_{-}: \bar{H} \otimes \bar{H} \rightarrow I \in$ Mor $\widehat{\mathcal{V}}$ called the $R$-matrices such that

$$
R_{+}=\left(\bar{H} \otimes \bar{H} \stackrel{\Omega}{\longrightarrow} \bar{H} \otimes \bar{H} \stackrel{R_{-}}{\longrightarrow} I\right),
$$

where $\Omega$ denotes the morphism $1^{1} \otimes\left(c^{32} \circ c^{23}\right) \otimes 1^{4}: H^{12} \otimes H^{34} \rightarrow H^{12} \otimes H^{34}$,

$$
\begin{aligned}
& \varepsilon=\left(\bar{H} \simeq I \otimes \bar{H} \stackrel{\bar{\eta} \otimes \bar{H}}{\longrightarrow} \bar{H} \otimes \bar{H} \stackrel{R_{+}}{\longrightarrow} I\right), \\
& \varepsilon=\left(\bar{H} \simeq \bar{H} \otimes I \stackrel{\bar{H} \otimes \bar{\eta}}{\longrightarrow} \bar{H} \otimes \bar{H} \stackrel{R_{+}}{\longrightarrow} I\right),
\end{aligned}
$$




$$
\begin{aligned}
& \left(\bar{H} \otimes \bar{H} \otimes \bar{H} \stackrel{\bar{m} \otimes \bar{H}}{\longrightarrow} \bar{H} \otimes \bar{H} \stackrel{R_{+}}{\longrightarrow} I\right) \\
& =(\bar{H} \otimes \bar{H} \otimes \bar{H} \stackrel{\bar{H} \otimes \bar{H} \otimes \bar{\Delta}}{\longrightarrow} \bar{H} \otimes \bar{H} \otimes \bar{H} \otimes \bar{H} \\
& \left.\stackrel{\bar{H} \otimes R_{+} \otimes \bar{H}}{\longrightarrow} \bar{H} \otimes I \otimes \bar{H} \simeq \bar{H} \otimes \bar{H} \stackrel{R_{+}}{\longrightarrow} I\right), \\
& \left(\bar{H} \otimes \bar{H} \otimes \bar{H} \stackrel{\bar{H} \otimes \bar{m}}{\longrightarrow} \bar{H} \otimes \bar{H} \stackrel{R_{-}}{\longrightarrow} I\right) \\
& =(\bar{H} \otimes \bar{H} \otimes \bar{H} \stackrel{\bar{\Delta} \otimes \bar{H} \otimes \bar{H}}{\longrightarrow} \bar{H} \otimes \bar{H} \otimes \bar{H} \otimes \bar{H} \\
& \left.\stackrel{\bar{H} \otimes c^{-1} \otimes \bar{H}}{\longrightarrow} \bar{H} \otimes \bar{H} \otimes \bar{H} \otimes \bar{H} \stackrel{R_{-} \otimes R_{-}}{\longrightarrow} I \otimes I \simeq I\right), \\
& \left.H_{1^{\prime} 1^{\prime \prime}} \otimes H_{1^{\prime \prime \prime} 1^{4}} \otimes H_{1^{5} 2^{\prime \prime}} \otimes H_{1^{6} 2^{\prime}} \stackrel{R_{+} \otimes m}{\longrightarrow} I_{1^{\prime}} \otimes H_{1^{\prime \prime} 2} \simeq H_{12}\right) \\
& =\left(H_{1^{\prime} 2^{\prime}} \otimes H_{1^{\prime \prime} 2^{\prime \prime}} \simeq H_{1^{\prime} 2^{\prime \prime}} \otimes I_{2^{\prime}} \otimes H_{1^{\prime \prime} 2^{\prime \prime \prime}} \stackrel{H \otimes \Delta_{1^{\prime \prime} 2^{\prime} 2^{\prime \prime \prime}}}{\longrightarrow} H_{1^{\prime} 2^{\prime \prime \prime}} \otimes H_{1^{\prime \prime} 2^{\prime}} \otimes H_{2^{\prime \prime} 2^{4}}\right. \\
& \stackrel{r_{H}^{-1} \otimes c_{2^{\prime \prime} 2^{\prime \prime \prime}}^{-1}}{\longrightarrow} H_{1^{\prime} 2^{\prime \prime \prime}} \otimes H_{1^{\prime \prime} 2^{\prime}} \otimes I_{2^{\prime \prime}} \otimes H_{2^{4} 2^{5}} \stackrel{\Delta_{1^{\prime} 2^{\prime \prime} 2^{\prime \prime \prime}} \otimes H \otimes H}{\longrightarrow} \\
& \left.H_{1^{\prime} 2^{\prime \prime}} \otimes H_{1^{\prime \prime} 2^{\prime}} \otimes H_{2^{\prime \prime \prime} 2^{4}} \otimes H_{2^{5} 2^{6}} \stackrel{m \otimes R_{+}}{\longrightarrow} H_{12^{\prime}} \otimes I_{2^{\prime \prime}} \simeq H_{12}\right) \text {. }
\end{aligned}
$$

It is easier to look at these conditions in graphical form. Equation (5.1.2a) becomes
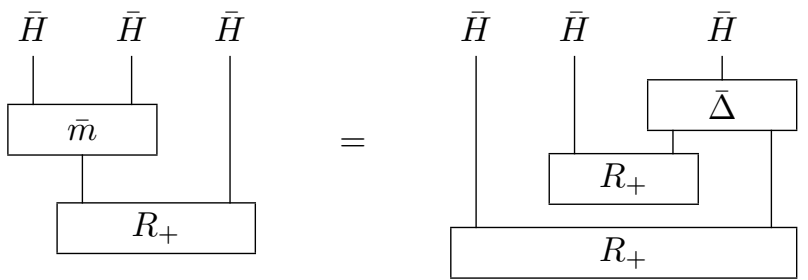

(5.1.2b) becomes

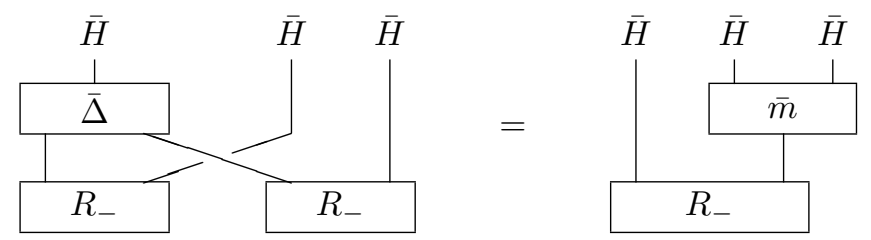

and (5.1.3) becomes

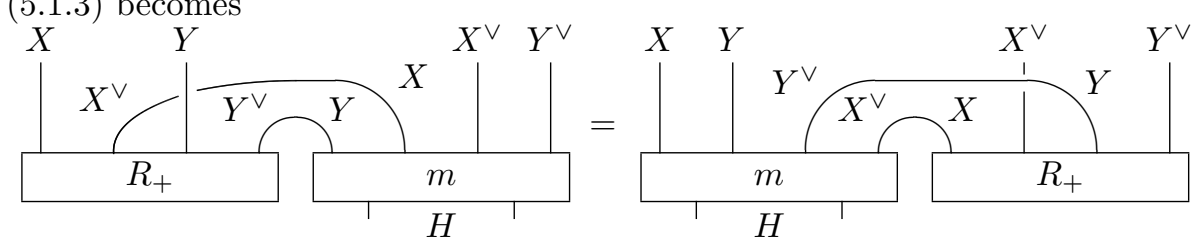

With a certain effort one can see that these properties are the dual ones to the equations for $R$-matrix written by Drinfeld [2].

5.1.2. THEOREM. Let $\left(H, R_{+}, R_{-}\right)$be a quasitriangular Hopf coalgebra. Then the categories of comodules ${ }^{H} \mathcal{V}$ and ${ }^{H} \widehat{\mathcal{V}}$ are braided and the braiding $R_{X, Y}: X \otimes Y \rightarrow Y \otimes X$ 
is given by

$$
\begin{aligned}
& R_{X, Y}=\left(X \otimes Y \stackrel{\bar{\delta}_{X} \otimes \bar{\delta}_{Y}}{\longrightarrow} \bar{H} \otimes X \otimes \bar{H} \otimes Y \underset{(432)_{+} \sim}{\sim} \bar{H} \otimes \bar{H} \otimes Y \otimes X\right. \\
&=\left(X \otimes Y \stackrel{R_{+} \otimes Y \otimes X}{\longrightarrow} I \otimes Y \otimes X \simeq Y \otimes X\right) \\
& \stackrel{\bar{\delta}_{X} \otimes \bar{\delta}_{Y}}{\longrightarrow} \bar{H} \otimes X \otimes \bar{H} \otimes Y \stackrel{(432)_{-} \sim}{\longrightarrow} \bar{H} \otimes \bar{H} \otimes Y \otimes X \\
&\left.\stackrel{R_{-} \otimes Y \otimes X}{\longrightarrow} I \otimes Y \otimes X \simeq Y \otimes X\right),
\end{aligned}
$$

or

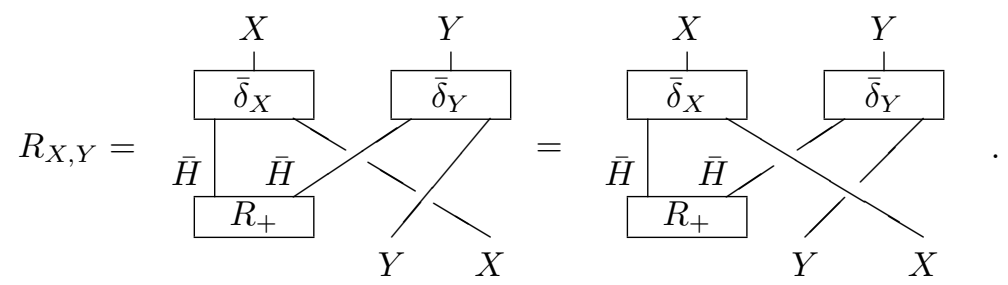

5.1.3. Remark. One can define quasitriangular bicoalgebras in the same way as in Definition 5.1.1, adding one condition - invertibility of (5.1.4).

5.1.4. Rem ark. Equation (5.1.3) can be written in 4 equivalent forms. By (5.1.1) one can replace $c^{-1}, R_{+}$by $c, R_{-}$in the left and/or, independently, in the right hand side of (5.1.3).

5.1.5. Proposition. Let $H$ be a bicoalgebra (e.g. a Hopf coalgebra). If ${ }^{H} \mathcal{V}$ has a braiding $R_{X, Y}: X \otimes Y \rightarrow Y \otimes X$, then $\left(H, R_{+}, R_{-}\right)$is quasitriangular, where $R_{+}, R_{-}$ are determined by

$$
\begin{aligned}
& \left(X \otimes X^{\vee} \otimes Y \otimes Y^{\vee} \stackrel{\ddot{i}_{X} \otimes \ddot{i}_{Y}}{\longrightarrow} \bar{H} \otimes \bar{H} \stackrel{R_{ \pm}}{\longrightarrow} I\right) \\
& =\left(X \otimes X^{\vee} \otimes Y \otimes Y^{\vee} \stackrel{X \otimes c^{ \pm 1} \otimes Y^{\vee}}{\longrightarrow} X \otimes Y \otimes X^{\vee} \otimes Y^{\vee} \stackrel{R_{X, Y} \otimes X^{\vee} \otimes Y^{\vee}}{\longrightarrow}\right. \\
& \left.Y \otimes X \otimes X^{\vee} \otimes Y^{\vee} \stackrel{Y \otimes \mathrm{ev} \otimes Y^{\vee}}{\longrightarrow} Y \otimes I \otimes Y^{\vee} \simeq Y \otimes Y^{\vee} \stackrel{\mathrm{ev}}{\longrightarrow} I\right) .
\end{aligned}
$$

5.2. Braiding for comodules over a braided Hopf algebra. It seems that there is no gadget, which would make the whole category of comodules over a braided Hopf algebra into a braided category. That is why the framework of braided Hopf algebras seems insufficient for the ideas like quantum braided groups. However, braided Hopf algebras, which came from quasitriangular squared Hopf coalgebras, make an exception.

Let $H$ be a quasitriangular Hopf coalgebra, and let $\bar{H}=\circledast H$. The category ${ }^{\bar{H}} \mathcal{V}$ of comodules over the braided Hopf algebra $\bar{H}$ is equivalent to the tensor product ${ }^{H} \mathcal{V} \otimes \mathcal{V}$ of two braided rigid monoidal categories by Theorem 2.6.1. This allows to introduce a braiding in $\bar{H} \mathcal{V}$.

Indeed, the tensor product

$$
\begin{aligned}
& \underline{\otimes}:\left({ }^{H} \mathcal{V} \otimes \mathcal{V}\right) \times\left({ }^{H} \mathcal{V} \otimes \mathcal{V}\right) \stackrel{\odot}{\rightarrow}{ }^{H} \mathcal{V} \otimes \mathcal{V} \otimes{ }^{H} \mathcal{V} \otimes \mathcal{V} \\
& \qquad \stackrel{1 \otimes P \otimes 1}{\longrightarrow}{ }^{H} \mathcal{V} \otimes{ }^{H} \mathcal{V} \otimes \mathcal{V} \otimes \mathcal{V} \stackrel{(\circledast \otimes \circledast)}{\longrightarrow} \mathcal{V} \otimes \mathcal{V}, \\
&(A \otimes B)_{12}=A_{1^{\prime} 2^{\prime} \otimes B_{1^{\prime \prime} 2^{\prime \prime}}} \text { for } A, B \in{ }^{H} \mathcal{V} \otimes \mathcal{V}, \\
&(M \odot X) \otimes(N \odot Y)=(M \otimes N) \odot(X \otimes Y) \text { for } M, N \in{ }^{H} \mathcal{V}, X, Y \in \mathcal{V},
\end{aligned}
$$

with the corresponding associativity and unit constraints makes $\left({ }^{H} \mathcal{V} \otimes \mathcal{V}, \underline{\otimes} I \odot I\right)$ into a monoidal category similarly to Section 1.5 . 
5.2.1. Proposition. The functorial isomorphism $\psi: A^{12} \otimes B^{34} \rightarrow A^{13} \otimes B^{24}, A, B \in$ $H \mathcal{V} \otimes$, determined by

$$
\psi=1 \otimes c \otimes 1: M \otimes X \otimes N \otimes Y \rightarrow M \otimes N \otimes X \otimes Y
$$

for $M, N \in{ }^{H} \mathcal{V}, X, Y \in \mathcal{V}$, gives a monoidal equivalence

$$
\left(\circledast, \psi, r_{I}^{-1}\right):\left({ }^{H} \mathcal{V} \otimes \mathcal{V}, \underline{\otimes}, I \odot I\right) \rightarrow\left({ }^{\bar{H}} \mathcal{V}, \otimes, I\right) .
$$

Each of the four isomorphisms

$$
\begin{aligned}
c_{ \pm \pm}:(M \odot X) \underline{\otimes}(N \odot Y)= & (M \otimes N) \odot(X \otimes Y) \\
& \stackrel{R^{ \pm 1} \odot c^{ \pm 1}}{\longrightarrow}(N \otimes M) \odot(Y \otimes X)=(N \odot Y) \underline{\otimes}(M \odot X)
\end{aligned}
$$

gives a braiding in $\left({ }^{H} \mathcal{V} \otimes \mathcal{V}, \underline{\otimes}, I \odot I\right)$. Thus, the category of $\bar{H}$-comodules is braided as well.

5.2.2. Corollary. The category $\left({ }^{\bar{H}} \mathcal{V}, \otimes, I\right)$ has braidings $c_{ \pm \pm}^{\prime}$ such that

$$
\begin{aligned}
c_{ \pm \pm}^{\prime}=((M \otimes X) \otimes(N \otimes Y) \stackrel{1 \otimes c \otimes 1}{\longrightarrow} M \otimes N \otimes X \otimes Y \\
\left.\stackrel{R^{ \pm 1} \otimes c^{ \pm 1}}{\longrightarrow} N \otimes M \otimes Y \otimes X \stackrel{1 \otimes c^{-1} \otimes 1}{\longrightarrow}(N \otimes Y) \otimes(M \otimes X)\right)
\end{aligned}
$$

for $M, N \in{ }^{H} \mathcal{V}, X, Y \in \mathcal{V}$.

6. Ribbon Hopf coalgebras. Let us assume that $V$ is braided. Fix the isomorphism $\zeta=u_{1}^{2}: X \rightarrow X^{\vee \vee}$. We want to discuss ribbon structures in the categories ${ }^{H} \mathcal{V}$. A ribbon structure in $\mathcal{V}$ is not required.

\subsection{Ribbon twists}

6.1.1. Definition. A ribbon Hopf coalgebra is a quasitriangular Hopf coalgebra $H$ equipped with a linear form $\Theta: \bar{H} \rightarrow I \in \widehat{\mathcal{V}}$ such that

$$
\begin{gathered}
(I \stackrel{\bar{\eta}}{\rightarrow} \bar{H} \stackrel{\Theta}{\longrightarrow} I)=\mathrm{id}_{I}, \\
\left(H^{12} \stackrel{c^{12}}{\longrightarrow} H^{21} \stackrel{\gamma_{\zeta}^{12}}{\longrightarrow} H^{12} \stackrel{\Theta}{\longrightarrow} I\right)=\Theta,
\end{gathered}
$$

$(\bar{H} \otimes \bar{H} \stackrel{\bar{\Delta} \otimes \bar{\Delta}}{\longrightarrow} \bar{H} \otimes \bar{H} \otimes \bar{H} \otimes \bar{H} \stackrel{\bar{\Delta} \otimes \Theta \otimes \bar{H} \otimes \Theta}{\longrightarrow} \bar{H} \otimes \bar{H} \otimes I \otimes \bar{H} \otimes I$

$$
\begin{aligned}
& \stackrel{\sim}{\longrightarrow} \bar{H} \otimes \bar{H} \otimes \bar{H} \stackrel{\bar{H} \otimes c}{\longrightarrow} \bar{H} \otimes \bar{H} \otimes \bar{H} \stackrel{\bar{H} \otimes \bar{\Delta} \otimes \bar{H}}{\longrightarrow} \\
& \left.\bar{H} \otimes \bar{H} \otimes \bar{H} \otimes \bar{H} \stackrel{R_{+} \otimes R_{-}}{\longrightarrow} I \otimes I \stackrel{\sim}{\longrightarrow} I\right)
\end{aligned}
$$

$$
=(\bar{H} \otimes \bar{H} \stackrel{\bar{m}}{\longrightarrow} \bar{H} \stackrel{\Theta}{\longrightarrow} I),
$$

$$
\begin{aligned}
& \left(H_{12} \stackrel{\sim}{\longrightarrow} H_{1^{\prime} 2} \otimes I_{1^{\prime \prime}} \stackrel{\Delta_{1^{\prime} 1^{\prime \prime} 2}}{\longrightarrow} H_{1^{\prime} 1^{\prime \prime}} \otimes H_{1^{\prime \prime \prime} 2} \stackrel{\Theta \otimes H}{\longrightarrow} I_{1^{\prime}} \otimes H_{1^{\prime \prime} 2} \stackrel{\sim}{\rightarrow} H_{12}\right) \\
& =\left(H_{12} \stackrel{\sim}{\longrightarrow} H_{12^{\prime \prime}} \otimes I_{2^{\prime}} \stackrel{\Delta_{12^{\prime} 2^{\prime \prime}}}{\longrightarrow} H_{12^{\prime}} \otimes H_{2^{\prime \prime} 2^{\prime \prime \prime}} \stackrel{H \otimes \Theta}{\longrightarrow} H_{12^{\prime}} \otimes I_{2^{\prime \prime}} \stackrel{\sim}{\longrightarrow} H_{12}\right)(6.1 .4)
\end{aligned}
$$

6.1.2. TheOREM. (a) The category ${ }^{H} \mathcal{V}$ of comodules over a quasitriangular Hopf coalgebra $H$ is ribbon if and only if $H$ is ribbon.

(b) A linear form $\Theta: \bar{H} \rightarrow I \in \widehat{\mathcal{V}}$ satisfying the equations (6.1.1)-(6.1.4) determines a ribbon twist in ${ }^{H} \mathcal{V}$ by the formula

$$
\theta_{X}=(X \stackrel{\bar{\delta}}{\rightarrow} \bar{H} \otimes X \stackrel{\Theta \otimes X}{\longrightarrow} I \otimes X \simeq X) \in \mathcal{V} .
$$


(c) A ribbon twist $\theta$ in ${ }^{H} \mathcal{V}$ determines the unique functional $\Theta$ with the properties (6.1.1)-(6.1.4) via the diagram

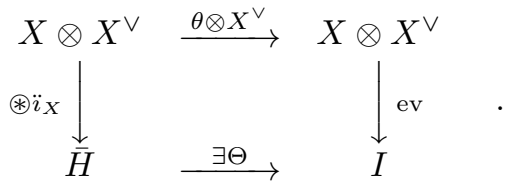

Acknowledgements. I would like to thank Yu. Bespalov and A. Sudbery for fruitful discussions and attention to this work.

\section{References}

[1] P. Deligne, Catégories tannakiennes, in: The Grothendieck Festschrift, Vol. II, Progress in Math. 87, Boston, Basel, Berlin: Birkhäuser, 1991, 111-195.

[2] V. G. Drinfeld, Quantum groups, Proceedings of the ICM, AMS, Providence, R.I. 1 (1987), $798-820$.

[3] A. Grothendieck and J. L. Verdier, Préfaisceuax, in: Théorie des topos et cohomologie étale des schémas (SGA 4), Lect. Notes Math. 269, Berlin, Heidelberg, New York: SpringerVerlag, 1972, 1-217.

[4] L. Hlavaty, Quantized braided groups, J. Math. Phys. 35 (1994), no. 5, 2560-2569.

[5] S. MacLane, Categories for the Working Mathematician, Springer-Verlag, 1971.

[6] B. Pareigis, Reconstruction of hidden symmetries, preprint 1994.

[7] N. Saavedra Rivano, Catégories Tannakiennes, Lect. Notes Math. 265, Berlin, Heidelberg, New York: Springer-Verlag, 1972.

[8] P. Schauenburg, Tannaka duality for Arbitrary Hopf Algebras, Algebra-Berichte 66 , München: R. Fisher, 1992 